CRYSTALLOGRAPHIC COMMUNICATIONS

ISSN 2056-9890

Received 19 April 2017

Accepted 2 July 2017

Edited by $\mathrm{H}$. Stoeckli-Evans, University of Neuchâtel, Switzerland

₹ Additional correspondence author, e-mail: elemam5@hotmail.com.

Keywords: crystal structure; 1,2,4-triazole-5thione; N-Mannich bases; adamantane; piperazine; biological activity; $\mathrm{C}-\mathrm{H} \ldots \mathrm{F}$ hydrogen bonding: $\mathrm{C}-\mathrm{H} \cdots \pi$ interactions.

CCDC references: 1559732; 1559731

Supporting information: this article has supporting information at journals.iucr.org/e

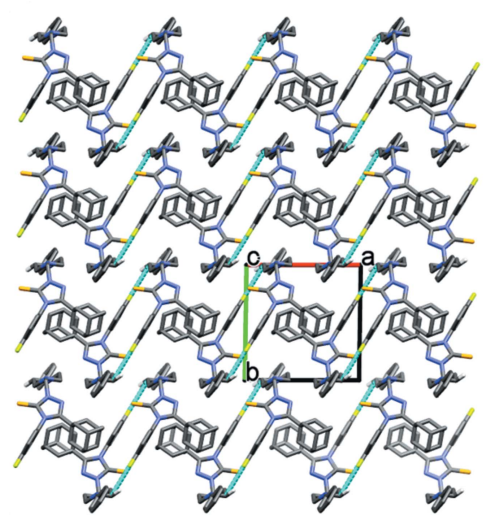

OPEN $\odot$ ACCESS

\section{Syntheses and crystal structures of two adamantyl- substituted 1,2,4-triazole-5-thione N-Mannich bases}

\author{
Monirah A. Al-Alshaikh, ${ }^{\text {a }}$ Aamal A. Al-Mutairi, ${ }^{a}$ Hazem A. Ghabbour, ${ }^{\text {b }}$ Ali A. \\ El-Emam, ${ }^{b} \neq$ Mohammed S. M. Abdelbaky ${ }^{c}$ and Santiago Garcia-Granda ${ }^{c} *$
}

\begin{abstract}
aDepartment of Chemistry, College of Sciences, King Saud University, Riyadh 11451, Saudi Arabia, ${ }^{\mathbf{b}}$ Department of Pharmaceutical Chemistry, College of Pharmacy, King Saud University, Riyadh 11451, Saudi Arabia, and ${ }^{\mathrm{C}}$ Department of Physical and Analytical Chemistry, Faculty of Chemistry, Oviedo University-CINN, Oviedo 33006, Spain.

*Correspondence e-mail: sgg@uniovi.es
\end{abstract}

In the title $N$-Mannich bases, 3-(adamantan-1-yl)-4-(4-fluorophenyl)-1[(4-phenylpiperazin-1-yl)methyl]-4,5-dihydro-1 H-1,2,4-triazole-5-thione $\left(\mathrm{C}_{29} \mathrm{H}_{34} \mathrm{FN}_{5} \mathrm{~S}\right)$ (I), and 3-(adamantan-1-yl)-4-(4-fluorophenyl)-1-\{[4-(2-methoxyphenyl)piperazin-1-yl]-methyl\}-4,5-dihydro-1H-1,2,4-triazole-5-thione $\left(\mathrm{C}_{30} \mathrm{H}_{36} \mathrm{FN}_{5} \mathrm{OS}\right)$ (II), fluorophenyl, adamantane and piperazine moieties are linked to a planar triazole ring. There is an additional phenyl ring on the piperazine ring in (I) and a methoxyphenyl ring in (II). In compound (I), the fluorophenyl and phenyl rings are inclined to the triazole ring by 86.55 (13) and $60.52(12)^{\circ}$, respectively, and the two aryl rings are inclined to one another by $66.37(13)^{\circ}$. In compound (II), the corresponding dihedral angles are 83.35 (13), 71.38 (15) and $11.97(16)^{\circ}$, respectively. The crystal structure of (I) shows pairs of $\mathrm{C}-\mathrm{H} \cdots \mathrm{F}$ hydrogen bonds forming inversion dimers, while in the crystal of compound (II), in addition to the $\mathrm{C}-\mathrm{H} \cdots \mathrm{F}$ hydrogen bonds that generate chains parallel to the $b$ axis, there are $\mathrm{C}-\mathrm{H} \cdots \pi$ interactions present that link the chains to form layers parallel to the $a b$ plane.

\section{Chemical context}

The incorporation of an adamantyl moiety into various bioactive molecules results in compounds with relatively high lipophilicity, which in turn modifies the bioavailability and modulates the therapeutic indices through various mechanisms (Liu et al., 2011; Lamoureux \& Artavia, 2010). Several adamantane-based drugs have been developed as antiviral (Davies et al., 1964; Togo et al., 1968; Rosenthal et al., 1982; ElEmam et al., 2004; Burstein et al., 1999; Balzarini et al., 2009), anti-Parkinsonian (Schwab et al., 1969) and hypoglycaemic (Villhauer et al., 2003; Augeri et al., 2005) drugs. In addition, numerous adamantane-based analogues have promising anticancer (Sun et al., 2002), bactericidal (Protopopova et al., 2005; El-Emam et al., 2013; Kadi et al., 2010; Al-Abdullah et al.; 2014; Al-Deeb et al., 2006) and fungicidal (Omar et al., 2010) activities. In a continuation of our ongoing studies on the pharmacological and structural properties of adamantyl 1,2,4triazole derivatives (Al-Abdullah et al., 2012; Al-Tamimi et al., 2014; El-Emam et al.; 2013; 2014), we report herein on the synthesis and crystal structures of the title adamantyl-substituted 1,2,4-triazole-5-thione $N$-Mannich bases, (I) and (II). 


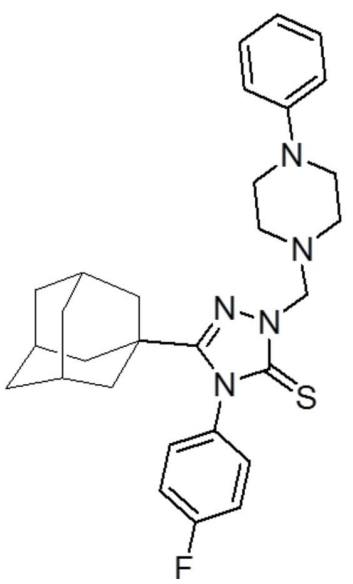

(I)

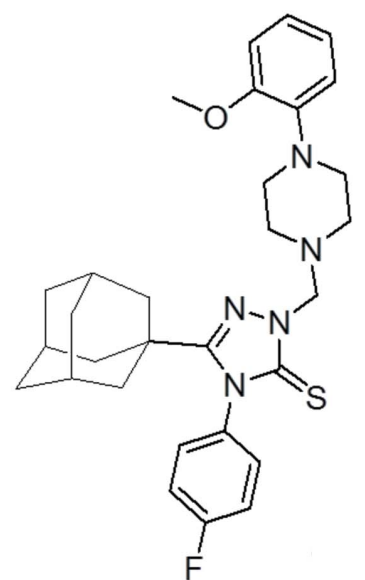

(II)

\section{Structural commentary}

The molecular structures of the title compounds, (I) and (II), are illustrated in Figs. 1 and 2, respectively. In both molecules the piperazine rings have a chair conformation, with the $\mathrm{N}$ bound substituents occupying equatorial positions. In (I), the fluorophenyl ring (C13-C18) and the phenyl ring (C24-C29) are inclined to the triazole ring $(\mathrm{N} 1-\mathrm{N} 3 / \mathrm{C} 11 / \mathrm{C} 12)$ by 86.55 (13) and $60.52(12)^{\circ}$, respectively. The two aryl rings are inclined to one another by 66.37 (13) ${ }^{\circ}$. In compound (II), the fluorophenyl ring (C13-C18) and the phenyl ring (C24-C29) are inclined to the triazole ring $(\mathrm{N} 1-\mathrm{N} 3 / \mathrm{C} 11 / \mathrm{C} 12)$ by $83.35(13)$ and $71.38(15)^{\circ}$, respectively, while the two aryl rings are inclined to one another by only $11.97(16)^{\circ}$. This difference in conformation is illustrated by the structural overlap diagram, shown in Fig. 3.

\section{Supramolecular features}

In the crystal of compound (I), molecules are linked by pairs of $\mathrm{C}-\mathrm{H} \cdots \mathrm{F}$ hydrogen bonds, forming inversion dimers (Table 1 and Fig. 4). In compound (II), molecules are linked by

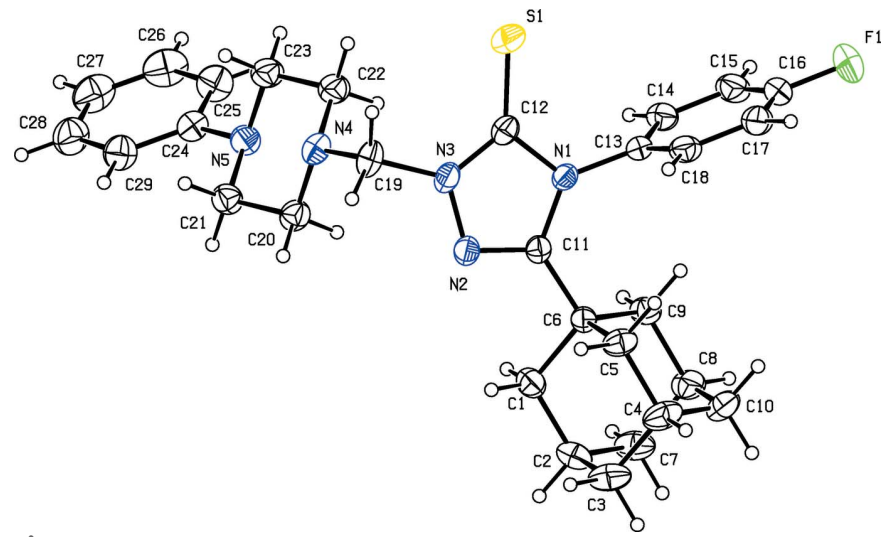

Figure 1

The molecular structure of compound (I), with the atom labelling and $30 \%$ probability displacement ellipsoids.

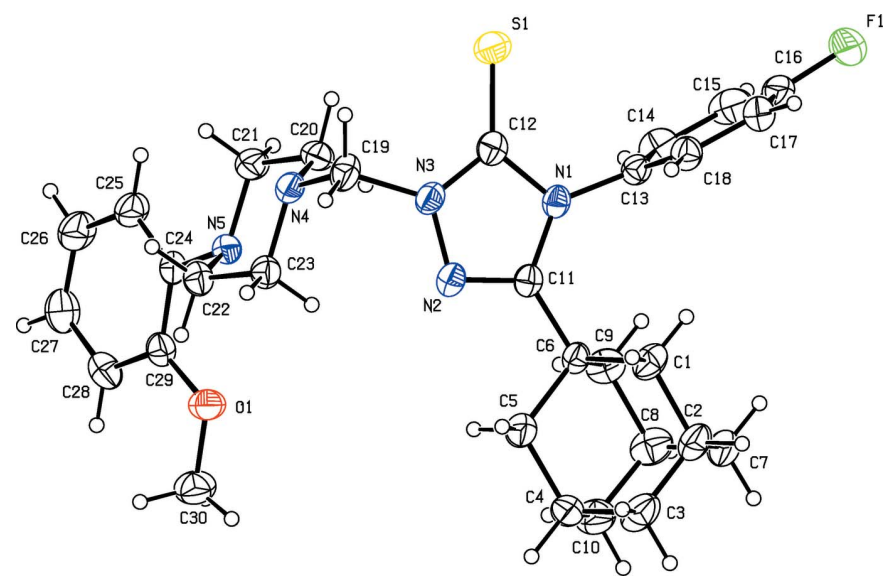

Figure 2

The molecular structure of compound (II), with the atom labelling and $30 \%$ probability displacement ellipsoids.

$\mathrm{C}-\mathrm{H} \cdots \mathrm{F}$ hydrogen bonds, forming chains parallel to the $b$ axis direction. The chains are linked by $\mathrm{C}-\mathrm{H} \cdots \pi$ interactions, forming layers parallel to the $a b$ plane (Table 2 and Fig. 5).

\section{Database survey}

A search of the Cambridge Structural Database (Version 5.38, last update May 2017; Groom et al., 2016) for the 3-(adamantan-1-yl)-4-[(piperazin-1-yl) methyl]-1,2,4-triazole3(3H)-thione moiety gave 14 hits. One compound, 3(adamantan-1-yl)-4-phenyl-1-[(4-phenylpiperazin-1-yl)methyl]-1H-1,2,4-triazole-5(4H)-thione (GAPWUR; Al-Abdullah et al., 2012), is very similar to compound (I). It has a phenyl ring substituent on the piperazine ring and a phenyl ring substituent on the triazole ring, which are inclined to one another by $72.4(2)^{\circ}$, and by $89.0(2)$ and $74.4(2)^{\circ}$, respectively, to the triazole ring. In compound (I), the corresponding dihedral angles are $66.37(13), 86.55(13)$ and $60.52(12)^{\circ}$, respectively. Two compounds have a 2-methoxyphenyl ring

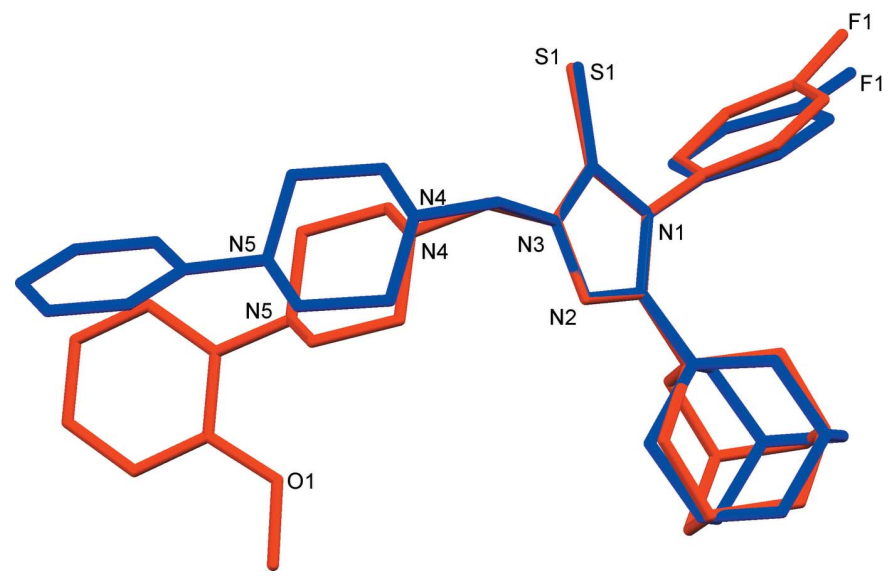

Figure 3

A structural overlap of compounds (I) and (II) [colour code: (I) blue, (II) red]. 
Table 1

Hydrogen-bond geometry $\left(\AA{ }^{\circ}\right)$ for $(\mathrm{I})$.

\begin{tabular}{lllll}
\hline$D-\mathrm{H} \cdots A$ & $D-\mathrm{H}$ & $\mathrm{H} \cdots A$ & $D \cdots A$ & $D-\mathrm{H} \cdots A$ \\
\hline $\mathrm{C} 22-\mathrm{H} 22 B \cdots \mathrm{F} 1^{\mathrm{i}}$ & 0.99 & 2.49 & $3.332(4)$ & 142 \\
\hline
\end{tabular}

Symmetry code: (i) $-x+2,-y+1,-z+1$.

Table 2

Hydrogen-bond geometry $\left(\AA{ }^{\circ}{ }^{\circ}\right)$ for (II).

$C g 1$ and $C g 8$ are the centroids of rings (N1-N3/C11/C12) and (C24-C29), respectively.

\begin{tabular}{lllll}
\hline$D-\mathrm{H} \cdots A$ & $D-\mathrm{H}$ & $\mathrm{H} \cdots A$ & $D \cdots A$ & $D-\mathrm{H} \cdots A$ \\
\hline $\mathrm{C} 21-\mathrm{H} 21 A \cdots \mathrm{F} 1^{\mathrm{i}}$ & 0.97 & 2.47 & $3.407(3)$ & 162 \\
$\mathrm{C} 18-\mathrm{H} 18 A \cdots C g 1^{\mathrm{ii}}$ & 0.93 & 2.81 & 3.661 & 152 \\
$\mathrm{C} 9-\mathrm{H} 9 A \cdots C g 8^{\mathrm{iii}}$ & 0.97 & 2.80 & 3.697 & 155 \\
\hline
\end{tabular}

Symmetry codes: (i) $x, y-1, z$; (ii) $-x,-y,-z$; (iii) $-x+1, y-\frac{1}{2},-z+\frac{1}{2}$.

substituent on the piperazine ring, viz. (3-(1-adamantyl)-1-\{[4(2-methoxyphenyl)piperazin-1-yl]methyl\}-4-methyl-1 $H$-1,2,4triazole-5(4H)-thione (YUPVIP; El-Emam et al., 2014), with a methyl substituent on the triazole ring, and 3-(adamantan-1yl)-4-ethyl-1-\{[4-(2-methoxyphenyl)piperazin-1-yl]methyl\}1H-1, 2,4-triazole-5(4H)-thione (RITMUE; Al-Tamimi et al., 2010) with an ethyl substituent on the triazole ring. In these two compounds the methoxyphenyl rings are inclined to the triazole ring by $67.1(1)$ and $59.4(1)^{\circ}$, respectively. In compound (II), the corresponding dihedral angle is $71.38(15)^{\circ}$.

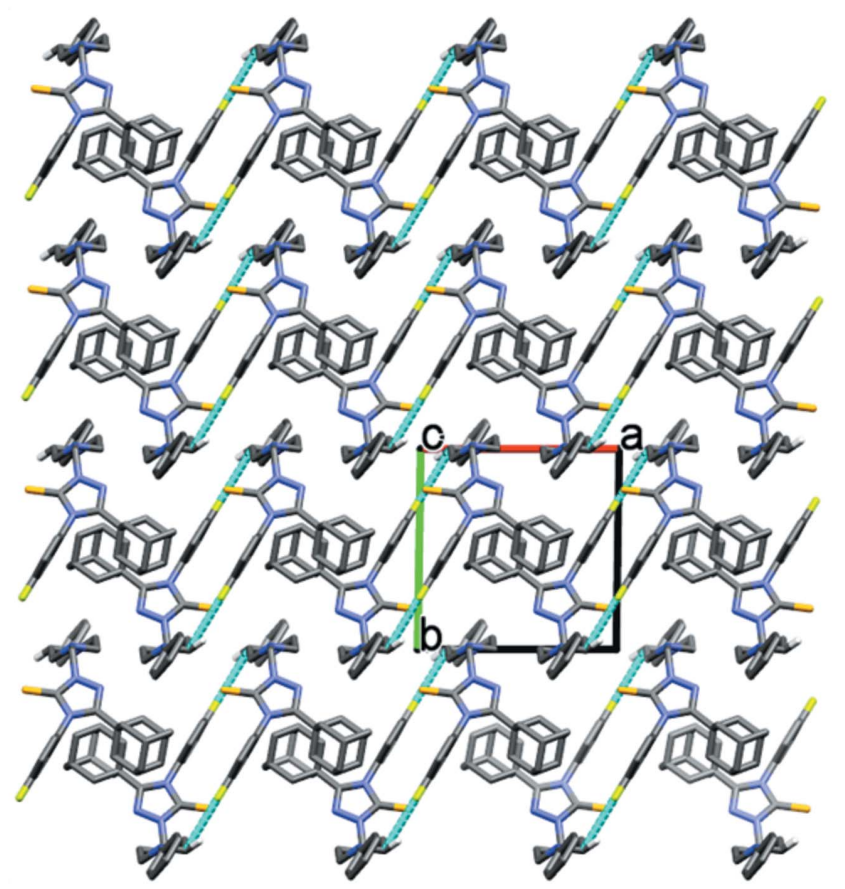

Figure 4

The crystal packing of compound (I), viewed along the $c$ axis. The hydrogen bonds are shown as dashed lines (see Table 1), and only the $\mathrm{H}$ atoms involved in these interactions have been included.

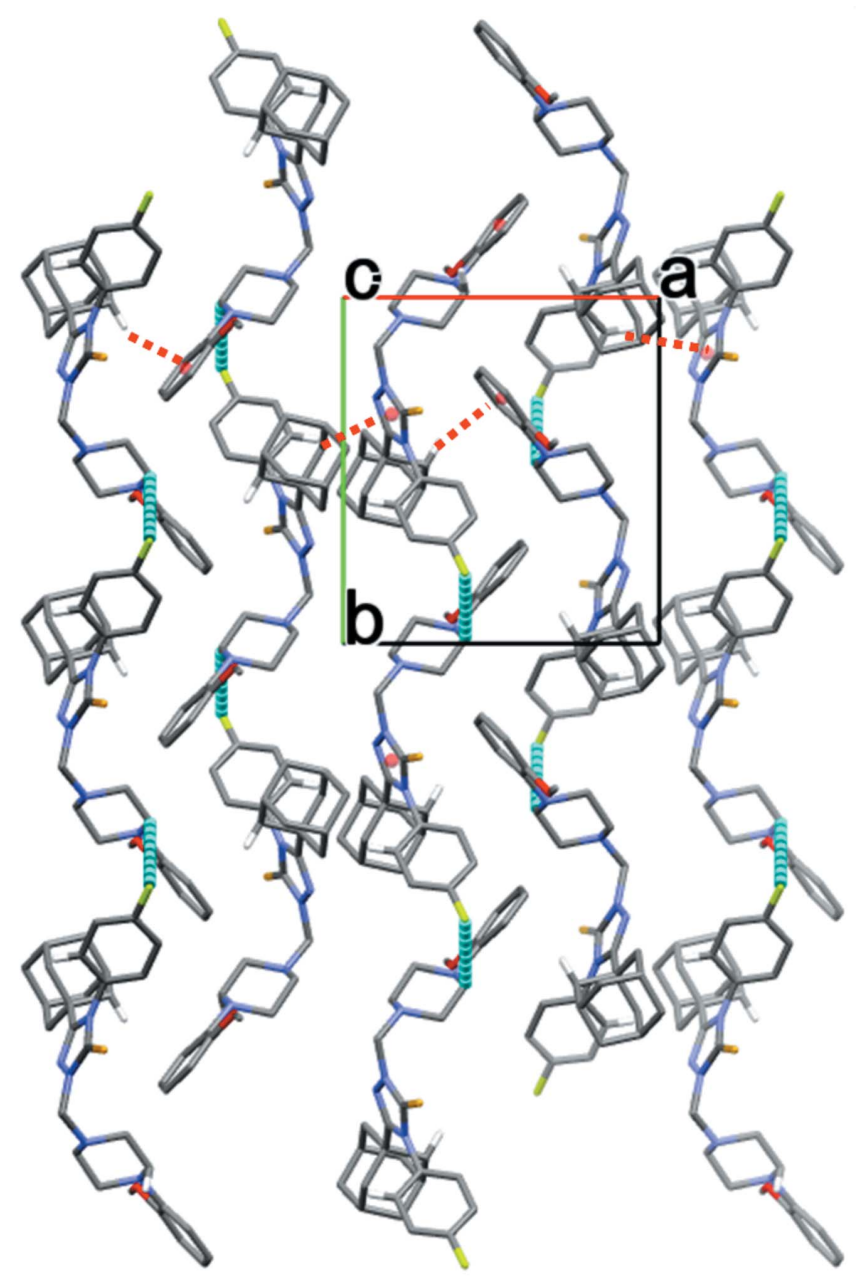

Figure 5

The crystal packing of compound (II), viewed along the $c$ axis, showing the $\mathrm{C}-\mathrm{H} \cdots \mathrm{F}$ hydrogen bonds (dashed cyan lines) and some of the $\mathrm{C}-$ $\mathrm{H} \cdots \pi$ interactions (dashed red lines); see Table 2. Only the $\mathrm{H}$ atoms involved in these interactions have been included.

\section{Synthesis and crystallization}

The title compounds, (I) and (II), were synthesized via the reaction of 3-(adamantan-1-yl)-4-(4-fluoromethyl)-4H-1,2,4triazole-5-thiol (Al-Deeb et al., 2006) with the appropriate monosubstituted piperazie and a formaldehyde solution, as outlined in Fig. 6.

Compound (I): 1-Phenylpiperazine ( $325 \mathrm{mg}, 2 \mathrm{mmol}$ ) and a $37 \%$ formaldehyde solution $(1 \mathrm{ml})$ were added to a solution of 3-(adamantan-1-yl)-4-(4-fluoromethyl)-4H-1,2,4-triazole-5thiol $(659 \mathrm{mg}, 2 \mathrm{mmol})$ in ethanol $(10 \mathrm{ml})$, and the mixture was heated under reflux for $1 \mathrm{~h}$ then allowed to stand overnight. Cold water $(3 \mathrm{ml})$ was slowly added and the mixture was stirred for $20 \mathrm{~min}$. The precipitated crude product was filtered, washed with water, dried, and crystallized from ethanol to yield $846 \mathrm{mg}(84 \%)$ of compound (I) as colourless plateshaped crystals (m.p. 469-471 K). ${ }^{1} \mathrm{H}$ NMR (700.17 MHz): $\delta$ 1.47-1.49 ( $m, 3 \mathrm{H}$, adamantane-H), 1.60-1.62 ( $m, 3 \mathrm{H}$, adamantane-H), $1.80(s, 6 \mathrm{H}$, adamantane- $\mathrm{H}), 1.89(s, 3 \mathrm{H}$, 

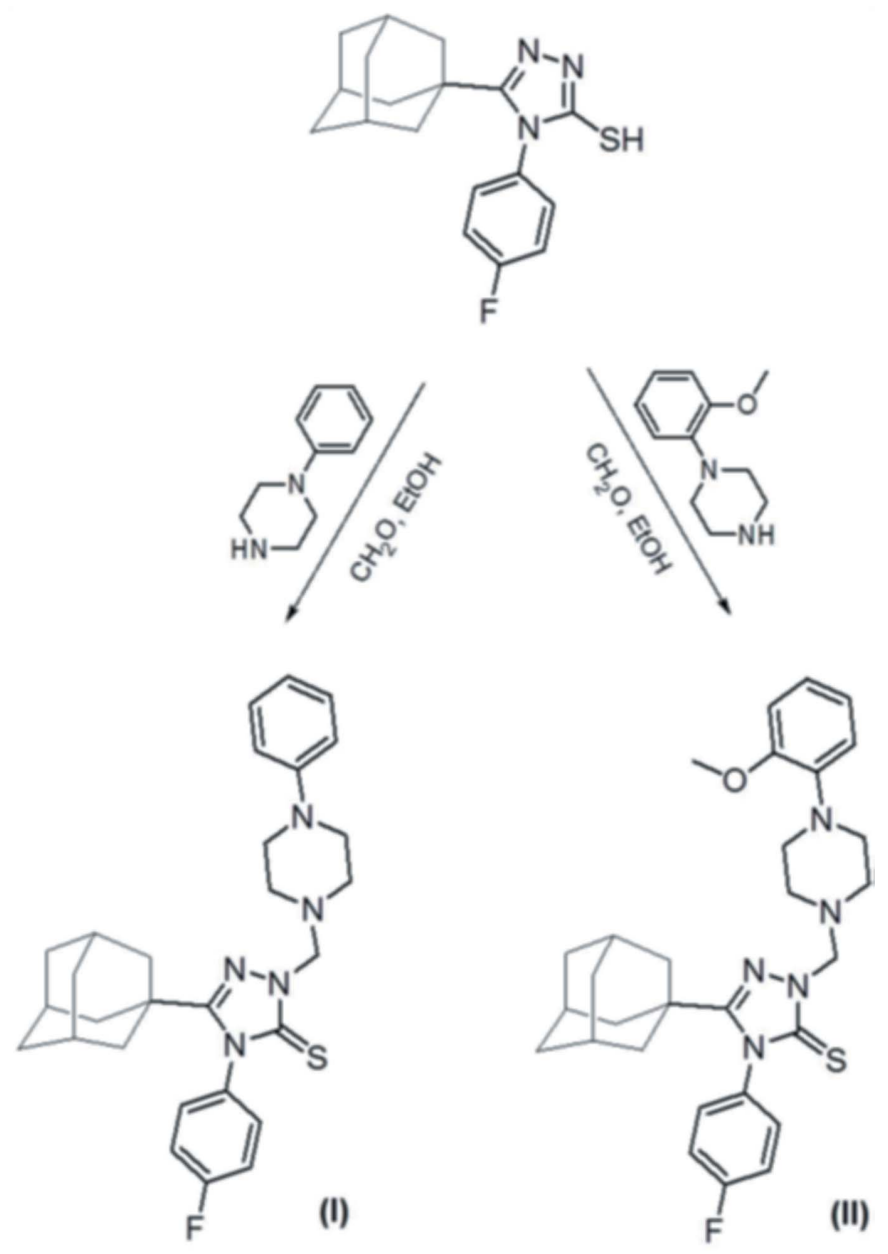

Figure 6

Reaction schemes for the syntheses of compounds (I) and (II).

adamantane-H), 2.89-2.91 ( $m, 4 \mathrm{H}$, piperazine-H), 3.14-3.15 $\left(m, 4 \mathrm{H}\right.$, piperazine-H), $5.14\left(s, 2 \mathrm{H}, \mathrm{CH}_{2}\right), 6.77-6.79(m, 1 \mathrm{H}$, Ar-H), 6.94 ( $d, 2 \mathrm{H}, \mathrm{Ar}-\mathrm{H}, J=8.4 \mathrm{~Hz}), 7.20-7.22(m, 2 \mathrm{H}, \mathrm{Ar}-\mathrm{H})$, 7.41-7.49 ( $m, 4 \mathrm{H}, \mathrm{Ar}-\mathrm{H}) .{ }^{13} \mathrm{C}$ NMR (125.76 MHz): $\delta$ 27.61, 36.07, 39.62, 39.74 (adamantane-C), 48.73, 50.30 (piperazineC), $69.06\left(\mathrm{CH}_{2}\right), 116.0,116.78,119.41,129.37,132.80,133.10$, 151.48, 156.28 (Ar-C), 162.17 (triazole C-3), $170.95(\mathrm{C}=\mathrm{S})$.

Compound (II): 1-(2-Methoxyphenyl)piperazine (385 mg, $2 \mathrm{mmol}$ ) and a $37 \%$ formaldehyde solution $(1 \mathrm{ml})$ were added to a solution of 3-(adamantan-1-yl)-4-(4-fluoromethyl)-4H1,2,4-triazole-5-thiol (659 mg, $2 \mathrm{mmol})$ in ethanol (10 ml), and the mixture was heated under reflux for $1 \mathrm{~h}$ then allowed to stand overnight. The precipitated crude product was filtered, washed with cold ethanol, dried, and crystallized from ethanol to yield $865 \mathrm{mg}$ (81\%) of compound (II) as colourless blocklike crystals (m.p. 462-464 K). ${ }^{1} \mathrm{H}$ NMR (700.17 MHz): $\delta 1.49-$ $1.50(m, 3 \mathrm{H}$, adamantane-H), 1.61-1.63 ( $m, 3 \mathrm{H}$, adamantane$\mathrm{H}), 1.83(s, 6 \mathrm{H}$, adamantane-H), $1.90(s, 3 \mathrm{H}$, adamantane- $\mathrm{H})$, 2.89-2.90 ( $m, 4 \mathrm{H}$, piperazine-H), 2.96-2.98 ( $m, 4 \mathrm{H}$, piperazine$\mathrm{H}), 3.78\left(\mathrm{~s}, 3 \mathrm{H}, \mathrm{OCH}_{3}\right), 5.11(\mathrm{~s}, 2 \mathrm{H}, \mathrm{CH} 2), 6.88-6.96(m, 4 \mathrm{H}$, Ar-H), 7.42-7.52 ( $m, 4 \mathrm{H}, \mathrm{Ar}-\mathrm{H}) .{ }^{13} \mathrm{C}$ NMR $(125.76 \mathrm{MHz}): \delta$ 27.61, 35.77, 36.07, 39.61 (adamantane-C), 50.54, 50.58 (piperazine-C), $55.65\left(\mathrm{OCH}_{3}\right), 69.39\left(\mathrm{CH}_{2}\right), 112.08,116.78$,
$118.51,121.23,123.02,132.79,133.13,141.53,152.36,156.32$ (Ar-C), 162.17 (triazole C-3), $171.0(\mathrm{C}=\mathrm{S})$.

Suitable single crystals of compounds (I) and (II) were obtained by slow evaporation of $\mathrm{CHCl}_{3}: \mathrm{EtOH}$ solutions (1:1, $5 \mathrm{ml}$ ) at room temperature.

\section{Refinement}

Crystal data, data collection and structure refinement details are summarized in Table 3.The C-bound $\mathrm{H}$ atoms were positioned geometrically and treated as riding atoms: $\mathrm{C}-\mathrm{H}=$ $0.93-1.00 \AA$ with $U_{\text {iso }}(\mathrm{H})=1.5 U_{\text {eq }}(\mathrm{C}$-methyl $)$ and $1.2 U_{\text {eq }}(\mathrm{C})$ for other $\mathrm{H}$ atoms.

\section{Acknowledgements}

The authors extend their appreciation to the Deanship of Scientific Research and the Research Centre, College of Sciences, King Saud University for funding this research.

\section{Funding information}

Financial support from the Spanish Ministerio de Economía y Competitividad (MINECO-13-MAT2013-40950-R, MAT2016-78155-C2-1-R and FPI grant BES-2011-046948 to MSMA), Gobierno del Principado de Asturias (GRUPIN14060) and FEDER funding, is also gratefully acknowledged

\section{References}

Al-Abdullah, E. S., Asiri, H. H., El-Emam, A. \& Ng, S. W. (2012). Acta Cryst. E68, 0345.

Al-Abdullah, E. S., Asiri, H. H., Lahsasni, S., Habib, E. E., Ibrahim, T. M. \& El-Emam, A. A. (2014). Drug Des. Dev. Ther. 8, 505-518.

Al-Deeb, O. A., Al-Omar, M. A., El-Brollosy, N. R., Habib, E. E., Ibrahim, T. M. \& El-Emam, A. A. (2006). Arzneim.-Forsch. Drug. Res. 56, 40-47.

Al-Tamimi, A.-M. S., Bari, A., Al-Omar, M. A., Alrashood, K. A. \& El-Emam, A. A. (2010). Acta Cryst. E66, o1756.

Al-Tamimi, A. S., El-Emam, A. A., Al-Deeb, O. A., Prasad, O., Pathak, S. K., Srivastava, R. \& Sinha, L. (2014). Spectrochim. Acta Part A, 124, 108-123.

Augeri, D. J., Robl, J. A., Betebenner, D. A., Magnin, D. R., Khanna, A., Robertson, J. G., Wang, A., Simpkins, L. M., Taunk, P., Huang, Q., Han, S., Abboa-Offei, B., Cap, M., Xin, L., Tao, L., Tozzo, E., Welzel, G. E., Egan, D. M., Marcinkeviciene, J., Chang, S. Y., Biller, S. A., Kirby, M. S., Parker, R. A. \& Hamann, L. G. (2005). J. Med. Chem. 48, 5025-5037.

Balzarini, J., Orzeszko-Krzesińska, B., Maurin, J. K. \& Orzeszko, A. (2009). Eur. J. Med. Chem. 44, 303-311.

Bruker (2012). APEX2, SAINT and SADABS. Bruker AXS Inc., Madison, Wisconsin, USA.

Burstein, M. E., Serbin, A. V., Khakhulina, T. V., Alymova, I. V., Stotskaya, L. L., Bogdan, O. P., Manukchina, E. E., Jdanov, V. V., Sharova, N. K. \& Bukrinskaya, A. G. (1999). Antiviral Res. 41, 135144.

Davies, W. L., Grunert, R. R., Haff, R. F., Mcgahen, J. W., Neumayer, E. M., Paulshock, M., Watts, J. C., Wood, T. R., Hermann, E. C. \& Hoffmann, C. E. (1964). Science, 144, 862-863.

Groom, C. R., Bruno, I. J., Lightfoot, M. P. \& Ward, S. C. (2016). Acta Cryst. B72, 171-179.

El-Emam, A. A., Al-Deeb, O. A., Al-Omar, M. A. \& Lehmann, J. (2004). Bioorg. Med. Chem. 12, 5107-5113. 
Table 3

Experimental details.

(I)

\section{Crystal data}

Chemical formula

$M_{\mathrm{r}}$

Crystal system, space group

Temperature (K)

$a, b, c(\AA)$

$\alpha, \beta, \gamma\left({ }^{\circ}\right)$

$V\left(\AA^{3}\right)$

$Z$

Radiation type

$\mu\left(\mathrm{mm}^{-1}\right)$

Crystal size (mm)

Data collection

Diffractometer

Absorption correction

$T_{\min }, T_{\max }$

No. of measured, independent and observed $[I>2 \sigma(I)]$ reflections

$R_{\text {int }}$

$(\sin \theta / \lambda)_{\max }\left(\AA^{-1}\right)$

Refinement

$R\left[F^{2}>2 \sigma\left(F^{2}\right)\right], w R\left(F^{2}\right), S$

No. of reflections

No. of parameters

$\mathrm{H}$-atom treatment

$\Delta \rho_{\max }, \Delta \rho_{\min }\left(\mathrm{e} \AA^{-3}\right)$

\section{$\mathrm{C}_{29} \mathrm{H}_{34} \mathrm{FN}_{5} \mathrm{~S}$}

503.67

Triclinic, $P \overline{1}$

296

10.4173 (5), 10.9849 (5),

$12.0002(6)$

72.769 (2), 84.623 (2), 89.244 (2)

$1305.66(11)$

Mo $K \alpha$

0.16

$0.45 \times 0.33 \times 0.09$

Bruker APEXII CCD

Multi-scan (SADABS; Bruker, 2012)

$0.939,0.986$

49580, 6011, 3533

0.076

0.650

$0.061,0.163,1.03$

6007

325

$\mathrm{H}$-atom parameters constrained

$0.26,-0.25$

(II)

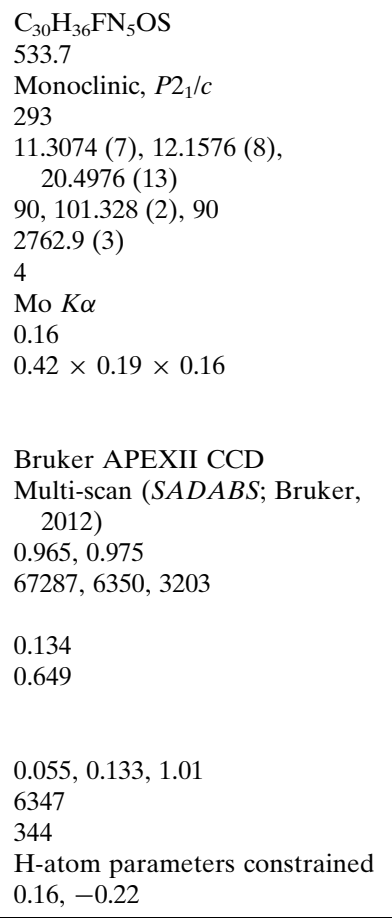

Computer programs: APEX2 and SAINT (Bruker, 2012), SHELXS2016 (Sheldrick, 2008), PLATON (Spek, 2009) and Mercury (Macrae et al., 2008), SHELXL2016 (Sheldrick, 2015), Win $G X$ (Farrugia, 2012) and publCIF (Westrip, 2010).

El-Emam, A. A., Al-Tamimi, A.-S., Al-Omar, M. A., Alrashood, K. A. \& Habib, E. E. (2013). Eur. J. Med. Chem. 68, 96-102.

El-Emam, A. A., Al-Tuwaijri, H. M., Al-Abdullah, E. S., Chidan Kumar, C. S. \& Fun, H.-K. (2014). Acta Cryst. E70, o25-o26.

Farrugia, L. J. (2012). J. Appl. Cryst. 45, 849-854.

Kadi, A. A., Al-Abdullah, E. S., Shehata, I. A., Habib, E. E., Ibrahim, T. M. \& El-Emam, A. A. (2010). Eur. J. Med. Chem. 45, 50065011.

Lamoureux, G. \& Artavia, G. (2010). Curr. Med. Chem. 17, 29672978.

Liu, J., Obando, D., Liao, V., Lifa, T. \& Codd, R. (2011). Eur. J. Med. Chem. 46, 1949-1963.

Macrae, C. F., Bruno, I. J., Chisholm, J. A., Edgington, P. R., McCabe, P., Pidcock, E., Rodriguez-Monge, L., Taylor, R., van de Streek, J. \& Wood, P. A. (2008). J. Appl. Cryst. 41, 466-470.

Omar, K., Geronikaki, A., Zoumpoulakis, P., Camoutsis, C., Soković, M., Cirić, A. \& Glamoćlija, J. (2010). Bioorg. Med. Chem. 18, 426432.
Protopopova, M., Hanrahan, C., Nikonenko, B., Samala, R., Chen, P., Gearhart, J., Einck, L. \& Nacy, C. A. (2005). J. Antimicrob. Chemother. 56, 968-974.

Rosenthal, K. S., Sokol, M. S., Ingram, R. L., Subramanian, R. \& Fort, R. C. (1982). Antimicrob. Agents Chemother. 22, 1031-1036.

Schwab, R. S., England, A. C. Jr, Poskanzer, D. C. \& Young, R. R. (1969). J. Am. Med. Assoc. 208, 1168-1170.

Sheldrick, G. M. (2008). Acta Cryst. A64, 112-122.

Sheldrick, G. M. (2015). Acta Cryst. C71, 3-8.

Spek, A. L. (2009). Acta Cryst. D65, 148-155.

Sun, S. Y., Yue, P., Chen, X., Hong, W. K. \& Lotan, R. (2002). Cancer Res. 62, 2430-2436.

Togo, Y., Hornick, R. B. \& Dawkins, A. T. (1968). J. Am. Med. Assoc. 203, 1089-1094.

Villhauer, E. B., Brinkman, J. A., Naderi, G. B., Burkey, B. F., Dunning, B. E., Prasad, K., Mangold, B. L., Russell, M. E. \& Hughes, T. E. (2003). J. Med. Chem. 46, 2774-2789.

Westrip, S. P. (2010). J. Appl. Cryst. 43, 920-925. 


\section{supporting information}

Acta Cryst. (2017). E73, 1135-1139 [https://doi.org/10.1107/S2056989017009756]

Syntheses and crystal structures of two adamantyl-substituted 1,2,4-triazole-5thione N-Mannich bases

Monirah A. Al-Alshaikh, Aamal A. Al-Mutairi, Hazem A. Ghabbour, Ali A. El-Emam, Mohammed S. M. Abdelbaky and Santiago Garcia-Granda

Computing details

For both structures, data collection: APEX2 (Bruker, 2012); cell refinement: SAINT (Bruker, 2012); data reduction: SAINT (Bruker, 2012); program(s) used to solve structure: SHELXS2016 (Sheldrick, 2008); program(s) used to refine structure: SHELXL2016 (Sheldrick, 2015); molecular graphics: PLATON (Spek, 2009) and Mercury (Macrae et al., 2008); software used to prepare material for publication: SHELXL2016 (Sheldrick, 2015), WinGX (Farrugia, 2012) and publCIF (Westrip, 2010).

3-(Adamantan-1-yl)-4-(4-fluorophenyl)-1-[(4-phenylpiperazin-1-yl)methyl]-4,5-dihydro-1 H-1,2,4-triazole-5thione (I)

Crystal data

$\mathrm{C}_{29} \mathrm{H}_{34} \mathrm{FN}_{5} \mathrm{~S}$

$M_{r}=503.67$

Triclinic, $P \overline{1}$

Hall symbol: -P 1

$a=10.4173(5) \AA$

$b=10.9849$ (5) $\AA$

$c=12.0002(6) \AA$

$\alpha=72.769(2)^{\circ}$

$\beta=84.623(2)^{\circ}$

$\gamma=89.244(2)^{\circ}$

$V=1305.66(11) \AA^{3}$

Data collection

Bruker APEXII CCD

diffractometer

Radiation source: fine-focus sealed tube

Graphite monochromator

$\varphi$ and $\omega$ scans

Absorption correction: multi-scan

(SADABS; Bruker, 2012)

$T_{\min }=0.939, T_{\max }=0.986$
$Z=2$

$F(000)=536$

$D_{\mathrm{x}}=1.281 \mathrm{Mg} \mathrm{m}^{-3}$

Mo $K \alpha$ radiation, $\lambda=0.71073 \AA$

Cell parameters from 9217 reflections

$\theta=2.5-23.7^{\circ}$

$\mu=0.16 \mathrm{~mm}^{-1}$

$T=296 \mathrm{~K}$

Plate, colourless

$0.45 \times 0.33 \times 0.09 \mathrm{~mm}$

49580 measured reflections

6011 independent reflections

3533 reflections with $I>2 \sigma(I)$

$R_{\text {int }}=0.076$

$\theta_{\max }=27.5^{\circ}, \theta_{\min }=2.5^{\circ}$

$h=-13 \rightarrow 13$

$k=-14 \rightarrow 14$

$l=-15 \rightarrow 15$ 


\section{Refinement}

Refinement on $F^{2}$

Least-squares matrix: full

$R\left[F^{2}>2 \sigma\left(F^{2}\right)\right]=0.061$

$w R\left(F^{2}\right)=0.163$

$S=1.03$

6007 reflections

325 parameters

0 restraints

0 constraints

Primary atom site location: structure-invariant direct methods
Secondary atom site location: difference Fourier map

Hydrogen site location: inferred from neighbouring sites

H-atom parameters constrained

$w=1 /\left[\sigma^{2}\left(F_{\mathrm{o}}^{2}\right)+(0.065 P)^{2}+0.6113 P\right]$ where $P=\left(F_{\mathrm{o}}{ }^{2}+2 F_{\mathrm{c}}{ }^{2}\right) / 3$

$(\Delta / \sigma)_{\max }<0.001$

$\Delta \rho_{\max }=0.26 \mathrm{e} \AA^{-3}$

$\Delta \rho_{\min }=-0.25$ e $\AA^{-3}$

Special details

Geometry. All esds (except the esd in the dihedral angle between two 1.s. planes) are estimated using the full covariance matrix. The cell esds are taken into account individually in the estimation of esds in distances, angles and torsion angles; correlations between esds in cell parameters are only used when they are defined by crystal symmetry. An approximate (isotropic) treatment of cell esds is used for estimating esds involving l.s. planes.

Refinement. Refinement of $\mathrm{F}^{2}$ against ALL reflections. The weighted R-factor $\mathrm{wR}$ and goodness of fit $\mathrm{S}$ are based on $\mathrm{F}^{2}$, conventional R-factors $R$ are based on $F$, with $F$ set to zero for negative $F^{2}$. The threshold expression of $F^{2}>2 \operatorname{sigma}\left(\mathrm{F}^{2}\right)$ is used only for calculating R-factors(gt) etc. and is not relevant to the choice of reflections for refinement. R-factors based on $\mathrm{F}^{2}$ are statistically about twice as large as those based on F, and R- factors based on ALL data will be even larger.

Fractional atomic coordinates and isotropic or equivalent isotropic displacement parameters $\left(\AA^{2}\right)$

\begin{tabular}{lllll}
\hline & $x$ & $y$ & $z$ & $U_{\text {iso }} * U_{\text {eq }}$ \\
\hline S1 & $0.97289(7)$ & $0.79726(7)$ & $0.53684(7)$ & $0.0643(2)$ \\
F1 & $0.9953(2)$ & $0.2584(2)$ & $0.8886(2)$ & $0.1173(9)$ \\
N1 & $0.74459(18)$ & $0.67026(17)$ & $0.62307(16)$ & $0.0391(5)$ \\
N2 & $0.59860(19)$ & $0.80635(18)$ & $0.54133(17)$ & $0.0454(5)$ \\
N3 & $0.7211(2)$ & $0.85604(18)$ & $0.50475(17)$ & $0.0472(5)$ \\
N4 & $0.7392(2)$ & $1.01606(19)$ & $0.30911(17)$ & $0.0494(5)$ \\
N5 & $0.7233(2)$ & $0.9884(2)$ & $0.08319(18)$ & $0.0497(5)$ \\
C1 & $0.3802(2)$ & $0.6521(3)$ & $0.6157(2)$ & $0.0528(7)$ \\
H1A & 0.3644 & 0.7428 & 0.6098 & $0.063^{*}$ \\
H1B & 0.3933 & 0.6444 & 0.5353 & $0.063^{*}$ \\
C2 & $0.2634(3)$ & $0.5709(3)$ & $0.6825(3)$ & $0.0592(7)$ \\
H2A & 0.1852 & 0.6003 & 0.64 & $0.071^{*}$ \\
C3 & $0.2423(3)$ & $0.5859(3)$ & $0.8050(3)$ & $0.0716(9)$ \\
H3A & 0.226 & 0.6764 & 0.7997 & $0.086^{*}$ \\
H3B & 0.1661 & 0.5346 & 0.8486 & $0.086^{*}$ \\
C4 & $0.3614(3)$ & $0.5414(3)$ & $0.8693(2)$ & $0.0660(8)$ \\
H4A & 0.3472 & 0.5516 & 0.9496 & $0.079^{*}$ \\
C5 & $0.4791(2)$ & $0.6218(3)$ & $0.8034(2)$ & $0.0514(7)$ \\
H5A & 0.5559 & 0.5932 & 0.8463 & $0.062^{*}$ \\
H5B & 0.4646 & 0.7123 & 0.7986 & $0.062^{*}$ \\
C6 & $0.5025(2)$ & $0.6083(2)$ & $0.67938(19)$ & $0.0365(5)$ \\
C7 & $0.2865(3)$ & $0.4317(3)$ & $0.6911(3)$ & $0.0669(8)$ \\
H7A & 0.3007 & 0.4216 & 0.6117 & $0.08^{*}$ \\
H7B & 0.2101 & 0.3795 & 0.7328 & $0.08^{*}$
\end{tabular}




\begin{tabular}{|c|c|c|c|c|}
\hline $\mathrm{C} 8$ & $0.4048(3)$ & $0.3872(3)$ & 0.7577 (3) & $0.0606(8)$ \\
\hline $\mathrm{H} 8 \mathrm{~A}$ & 0.4197 & 0.2952 & 0.7647 & $0.073^{*}$ \\
\hline C9 & $0.5233(2)$ & $0.4665(2)$ & $0.6893(2)$ & 0.0487 (6) \\
\hline H9A & 0.5367 & 0.4557 & 0.61 & $0.058^{*}$ \\
\hline H9B & 0.6011 & 0.4362 & 0.7303 & $0.058^{*}$ \\
\hline $\mathrm{C} 10$ & $0.3844(3)$ & $0.4035(3)$ & 0.8797 (3) & 0.0727 (9) \\
\hline $\mathrm{H} 10 \mathrm{~A}$ & 0.3093 & 0.3513 & 0.9245 & $0.087^{*}$ \\
\hline H10B & 0.4614 & 0.3744 & 0.9223 & $0.087 *$ \\
\hline C11 & $0.6145(2)$ & $0.6934(2)$ & $0.61309(19)$ & $0.0381(5)$ \\
\hline $\mathrm{C} 12$ & $0.8141(2)$ & $0.7769(2)$ & $0.5529(2)$ & $0.0442(6)$ \\
\hline $\mathrm{C} 13$ & $0.8086(2)$ & $0.5622(2)$ & $0.6933(2)$ & $0.0393(5)$ \\
\hline $\mathrm{C} 14$ & $0.8596(2)$ & $0.4721(2)$ & $0.6427(2)$ & $0.0505(6)$ \\
\hline H14A & 0.8511 & 0.4817 & 0.5624 & $0.061^{*}$ \\
\hline C15 & $0.9223(3)$ & $0.3690(3)$ & $0.7086(3)$ & $0.0648(8)$ \\
\hline H15A & 0.9572 & 0.3058 & 0.6753 & $0.078^{*}$ \\
\hline C16 & $0.9332(3)$ & $0.3597(3)$ & $0.8221(3)$ & $0.0700(9)$ \\
\hline $\mathrm{C} 17$ & 0.8867 (3) & $0.4476(3)$ & $0.8746(2)$ & $0.0679(9)$ \\
\hline H17A & 0.897 & 0.4375 & 0.9546 & $0.081 *$ \\
\hline C18 & $0.8236(2)$ & $0.5524(3)$ & 0.8079 (2) & $0.0520(7)$ \\
\hline H18A & 0.7913 & 0.6164 & 0.8412 & $0.062 *$ \\
\hline C19 & $0.7401(3)$ & $0.9915(2)$ & $0.4322(2)$ & 0.0548 (7) \\
\hline H19A & 0.6716 & 1.0425 & 0.459 & $0.066^{*}$ \\
\hline H19B & 0.8236 & 1.0223 & 0.4476 & $0.066^{*}$ \\
\hline $\mathrm{C} 20$ & $0.6161(3)$ & $1.0001(3)$ & 0.2677 (2) & $0.0530(7)$ \\
\hline $\mathrm{H} 20 \mathrm{~A}$ & 0.5484 & 1.0444 & 0.3039 & $0.064 *$ \\
\hline $\mathrm{H} 20 \mathrm{~B}$ & 0.5925 & 0.9084 & 0.2908 & $0.064 *$ \\
\hline $\mathrm{C} 21$ & $0.6245(3)$ & $1.0548(3)$ & $0.1353(2)$ & $0.0530(7)$ \\
\hline $\mathrm{H} 21 \mathrm{~A}$ & 0.5403 & 1.0445 & 0.1069 & $0.064 *$ \\
\hline H21B & 0.6462 & 1.1469 & 0.1121 & $0.064^{*}$ \\
\hline $\mathrm{C} 23$ & 0.8397 (3) & $0.9514(3)$ & $0.2571(2)$ & $0.0574(7)$ \\
\hline $\mathrm{H} 23 \mathrm{~A}$ & 0.8203 & 0.8588 & 0.2814 & $0.069 *$ \\
\hline $\mathrm{H} 23 \mathrm{~B}$ & 0.9236 & 0.9644 & 0.2848 & $0.069 *$ \\
\hline $\mathrm{C} 22$ & 0.8475 (3) & $1.0037(3)$ & $0.1250(2)$ & $0.0585(7)$ \\
\hline $\mathrm{H} 22 \mathrm{~A}$ & 0.8722 & 1.0951 & 0.1006 & $0.07^{*}$ \\
\hline H22B & 0.9145 & 0.958 & 0.0897 & $0.07 *$ \\
\hline $\mathrm{C} 24$ & 0.7295 (3) & $1.0128(2)$ & $-0.0403(2)$ & $0.0502(6)$ \\
\hline $\mathrm{C} 25$ & $0.8004(3)$ & $0.9305(3)$ & $-0.0903(3)$ & $0.0678(8)$ \\
\hline $\mathrm{H} 25 \mathrm{~A}$ & 0.8423 & 0.8599 & -0.0417 & $0.081^{*}$ \\
\hline $\mathrm{C} 26$ & 0.8102 (4) & 0.9509 (4) & $-0.2097(3)$ & $0.0820(10)$ \\
\hline $\mathrm{H} 26 \mathrm{~A}$ & 0.8584 & 0.8938 & -0.2426 & $0.098^{*}$ \\
\hline $\mathrm{C} 27$ & $0.7515(4)$ & $1.0521(4)$ & $-0.2813(3)$ & 0.0837 (11) \\
\hline $\mathrm{H} 27 \mathrm{~A}$ & 0.7599 & 1.066 & -0.3637 & $0.1^{*}$ \\
\hline $\mathrm{C} 28$ & $0.6804(3)$ & $1.1335(4)$ & $-0.2340(3)$ & $0.0782(10)$ \\
\hline $\mathrm{H} 28 \mathrm{~A}$ & 0.6387 & 1.2036 & -0.2835 & $0.094 *$ \\
\hline C29 & $0.6692(3)$ & $1.1139(3)$ & $-0.1138(3)$ & $0.0633(8)$ \\
\hline H29A & 0.6195 & 1.1706 & -0.0816 & $0.076^{*}$ \\
\hline
\end{tabular}


Atomic displacement parameters $\left(\AA^{2}\right)$

\begin{tabular}{|c|c|c|c|c|c|c|}
\hline & $U^{11}$ & $U^{22}$ & $U^{33}$ & $U^{12}$ & $U^{13}$ & $U^{23}$ \\
\hline $\mathrm{S} 1$ & $0.0496(4)$ & $0.0660(5)$ & $0.0691(5)$ & $-0.0162(3)$ & 0.0024 & $-0.0092(4)$ \\
\hline $\mathrm{F} 1$ & $0.0819(14)$ & $0.1034(16)$ & $0.1216(18)$ & $0.0377(12)$ & $-0.0074(12)$ & $0.0334(13)$ \\
\hline N1 & $0.0401(11)$ & $0.0368(11)$ & $0.0371(11)$ & $-0.0016(8)$ & $0.0025(8)$ & $-0.0076(8)$ \\
\hline $\mathrm{N} 2$ & $0.0497(12)$ & $0.0399(11)$ & $0.0410(11)$ & $0.0049(9)$ & $0.0022(9)$ & -0.0058 (9) \\
\hline N3 & $0.0543(13)$ & 0.0385 (11) & $0.0415(12)$ & $-0.0042(10)$ & $0.0042(9)$ & $-0.0030(9)$ \\
\hline N4 & $0.0572(13)$ & $0.0475(12)$ & $0.0411(12)$ & $-0.0079(10)$ & $-0.0001(10)$ & $-0.0107(9)$ \\
\hline N5 & $0.0502(13)$ & $0.0550(13)$ & 0.0439 (12) & $0.0054(10)$ & $-0.0022(9)$ & $-0.0155(10)$ \\
\hline $\mathrm{C} 1$ & $0.0481(15)$ & $0.0545(16)$ & $0.0541(16)$ & 0.0109 (12) & $-0.0079(12)$ & $-0.0130(13)$ \\
\hline $\mathrm{C} 2$ & 0.0377 (14) & 0.0703 (19) & $0.074(2)$ & $0.0088(13)$ & $-0.0132(13)$ & $-0.0255(15)$ \\
\hline $\mathrm{C} 3$ & 0.0447 (16) & $0.090(2)$ & $0.085(2)$ & $-0.0052(15)$ & $0.0155(15)$ & -0.0409 (18) \\
\hline $\mathrm{C} 4$ & $0.0536(17)$ & $0.098(2)$ & $0.0467(16)$ & $-0.0167(16)$ & $0.0137(13)$ & $-0.0262(16)$ \\
\hline $\mathrm{C} 5$ & $0.0447(14)$ & $0.0666(17)$ & $0.0468(15)$ & $-0.0077(12)$ & $0.0044(11)$ & $-0.0248(13)$ \\
\hline C6 & $0.0380(12)$ & $0.0370(12)$ & $0.0341(12)$ & $0.0051(10)$ & $0.0005(9)$ & $-0.0114(10)$ \\
\hline $\mathrm{C} 7$ & $0.0460(16)$ & $0.075(2)$ & $0.089(2)$ & $-0.0099(14)$ & $-0.0048(15)$ & $-0.0379(17)$ \\
\hline $\mathrm{C} 8$ & $0.0475(15)$ & $0.0402(15)$ & $0.093(2)$ & $-0.0066(12)$ & $-0.0009(14)$ & $-0.0186(14)$ \\
\hline C9 & 0.0415 (14) & $0.0434(14)$ & 0.0637 (17) & $0.0020(11)$ & $-0.0014(12)$ & $-0.0206(12)$ \\
\hline $\mathrm{C} 10$ & $0.0544(18)$ & $0.081(2)$ & $0.064(2)$ & $-0.0212(16)$ & $0.0055(14)$ & 0.0049 (16) \\
\hline $\mathrm{C} 11$ & $0.0438(13)$ & $0.0378(13)$ & $0.0320(12)$ & $0.0050(10)$ & $0.0003(10)$ & $-0.0107(10)$ \\
\hline $\mathrm{C} 12$ & $0.0507(15)$ & $0.0422(14)$ & $0.0381(13)$ & $-0.0036(11)$ & $0.0031(11)$ & $-0.0114(11)$ \\
\hline $\mathrm{C} 13$ & $0.0324(12)$ & $0.0402(13)$ & $0.0413(14)$ & $-0.0017(10)$ & $0.0008(10)$ & $-0.0073(10)$ \\
\hline $\mathrm{C} 14$ & $0.0423(14)$ & $0.0535(16)$ & $0.0567(16)$ & 0.0025 (12) & $-0.0017(12)$ & $-0.0185(13)$ \\
\hline $\mathrm{C} 15$ & $0.0451(16)$ & $0.0540(18)$ & $0.092(2)$ & $0.0079(13)$ & $-0.0001(15)$ & $-0.0185(16)$ \\
\hline $\mathrm{C} 16$ & $0.0418(16)$ & $0.066(2)$ & $0.079(2)$ & $0.0101(14)$ & $0.0003(15)$ & $0.0128(17)$ \\
\hline $\mathrm{C} 17$ & $0.0473(16)$ & $0.099(2)$ & $0.0415(16)$ & $0.0024(16)$ & $-0.0036(12)$ & $0.0037(16)$ \\
\hline C18 & $0.0403(14)$ & $0.0688(18)$ & $0.0453(15)$ & $-0.0011(12)$ & $0.0010(11)$ & $-0.0160(13)$ \\
\hline C19 & 0.0785 (19) & $0.0372(14)$ & $0.0445(15)$ & $-0.0067(13)$ & $-0.0011(13)$ & $-0.0069(11)$ \\
\hline $\mathrm{C} 20$ & $0.0562(16)$ & $0.0494(15)$ & $0.0514(16)$ & $0.0017(12)$ & $0.0045(12)$ & $-0.0148(12)$ \\
\hline $\mathrm{C} 21$ & $0.0516(15)$ & $0.0584(16)$ & $0.0490(16)$ & 0.0068 (13) & $-0.0034(12)$ & $-0.0164(13)$ \\
\hline $\mathrm{C} 23$ & $0.0482(15)$ & 0.0715 (19) & 0.0477 (16) & $-0.0004(13)$ & $-0.0034(12)$ & $-0.0105(13)$ \\
\hline $\mathrm{C} 22$ & $0.0480(16)$ & $0.076(2)$ & 0.0475 (16) & $0.0016(14)$ & $-0.0001(12)$ & $-0.0136(14)$ \\
\hline $\mathrm{C} 24$ & $0.0522(15)$ & $0.0520(16)$ & $0.0479(15)$ & $-0.0042(12)$ & $-0.0024(12)$ & $-0.0176(12)$ \\
\hline $\mathrm{C} 25$ & $0.076(2)$ & $0.069(2)$ & $0.063(2)$ & $0.0030(16)$ & $-0.0014(16)$ & $-0.0289(16)$ \\
\hline $\mathrm{C} 26$ & $0.084(2)$ & $0.103(3)$ & $0.073(2)$ & $-0.006(2)$ & $0.0066(19)$ & $-0.051(2)$ \\
\hline $\mathrm{C} 27$ & $0.086(2)$ & $0.117(3)$ & $0.0518(19)$ & $-0.022(2)$ & $-0.0013(18)$ & $-0.031(2)$ \\
\hline $\mathrm{C} 28$ & $0.079(2)$ & $0.094(3)$ & $0.056(2)$ & $-0.0078(19)$ & $-0.0103(17)$ & $-0.0124(18)$ \\
\hline $\mathrm{C} 29$ & 0.0690 (19) & 0.0678 (19) & $0.0543(18)$ & $0.0011(15)$ & $-0.0054(14)$ & $-0.0200(15)$ \\
\hline
\end{tabular}

Geometric parameters $\left(\AA,{ }^{\circ}\right)$

\begin{tabular}{llll}
\hline $\mathrm{S} 1-\mathrm{C} 12$ & $1.658(3)$ & $\mathrm{C} 8-\mathrm{H} 8 \mathrm{~A}$ & 1 \\
$\mathrm{~F} 1-\mathrm{C} 16$ & $1.358(3)$ & $\mathrm{C} 9-\mathrm{H} 9 \mathrm{~A}$ & 0.99 \\
$\mathrm{~N} 1-\mathrm{C} 11$ & $1.385(3)$ & $\mathrm{C} 9-\mathrm{H} 9 \mathrm{~B}$ & 0.99 \\
$\mathrm{~N} 1-\mathrm{C} 12$ & $1.389(3)$ & $\mathrm{C} 10-\mathrm{H} 10 \mathrm{~A}$ & 0.99 \\
$\mathrm{~N} 1-\mathrm{C} 13$ & $1.433(3)$ & $\mathrm{C} 10-\mathrm{H} 10 \mathrm{~B}$ & 0.99 \\
$\mathrm{~N} 2-\mathrm{C} 11$ & $1.303(3)$ & $\mathrm{C} 13-\mathrm{C} 18$ & $1.371(3)$
\end{tabular}




\begin{tabular}{|c|c|c|c|}
\hline $\mathrm{N} 2-\mathrm{N} 3$ & $1.375(3)$ & $\mathrm{C} 13-\mathrm{C} 14$ & $1.383(3)$ \\
\hline $\mathrm{N} 3-\mathrm{C} 12$ & $1.346(3)$ & $\mathrm{C} 14-\mathrm{C} 15$ & $1.373(4)$ \\
\hline N3-C19 & $1.490(3)$ & $\mathrm{C} 14-\mathrm{H} 14 \mathrm{~A}$ & 0.95 \\
\hline $\mathrm{N} 4-\mathrm{C} 19$ & $1.422(3)$ & $\mathrm{C} 15-\mathrm{C} 16$ & $1.351(5)$ \\
\hline $\mathrm{N} 4-\mathrm{C} 20$ & $1.449(3)$ & $\mathrm{C} 15-\mathrm{H} 15 \mathrm{~A}$ & 0.95 \\
\hline $\mathrm{N} 4-\mathrm{C} 23$ & $1.452(3)$ & $\mathrm{C} 16-\mathrm{C} 17$ & $1.361(5)$ \\
\hline N5-C24 & $1.421(3)$ & $\mathrm{C} 17-\mathrm{C} 18$ & $1.389(4)$ \\
\hline $\mathrm{N} 5-\mathrm{C} 21$ & $1.453(3)$ & C17-H17A & 0.95 \\
\hline N5-C22 & $1.459(3)$ & $\mathrm{C} 18-\mathrm{H} 18 \mathrm{~A}$ & 0.95 \\
\hline $\mathrm{C} 1-\mathrm{C} 2$ & $1.530(4)$ & C19-H19A & 0.99 \\
\hline $\mathrm{C} 1-\mathrm{C} 6$ & $1.545(3)$ & C19-H19B & 0.99 \\
\hline $\mathrm{C} 1-\mathrm{H} 1 \mathrm{~A}$ & 0.99 & $\mathrm{C} 20-\mathrm{C} 21$ & $1.518(3)$ \\
\hline $\mathrm{C} 1-\mathrm{H} 1 \mathrm{~B}$ & 0.99 & $\mathrm{C} 20-\mathrm{H} 20 \mathrm{~A}$ & 0.99 \\
\hline $\mathrm{C} 2-\mathrm{C} 7$ & $1.519(4)$ & $\mathrm{C} 20-\mathrm{H} 20 \mathrm{~B}$ & 0.99 \\
\hline $\mathrm{C} 2-\mathrm{C} 3$ & $1.523(4)$ & $\mathrm{C} 21-\mathrm{H} 21 \mathrm{~A}$ & 0.99 \\
\hline $\mathrm{C} 2-\mathrm{H} 2 \mathrm{~A}$ & 1 & $\mathrm{C} 21-\mathrm{H} 21 \mathrm{~B}$ & 0.99 \\
\hline $\mathrm{C} 3-\mathrm{C} 4$ & $1.521(4)$ & $\mathrm{C} 23-\mathrm{C} 22$ & $1.513(4)$ \\
\hline $\mathrm{C} 3-\mathrm{H} 3 \mathrm{~A}$ & 0.99 & $\mathrm{C} 23-\mathrm{H} 23 \mathrm{~A}$ & 0.99 \\
\hline $\mathrm{C} 3-\mathrm{H} 3 \mathrm{~B}$ & 0.99 & $\mathrm{C} 23-\mathrm{H} 23 \mathrm{~B}$ & 0.99 \\
\hline $\mathrm{C} 4-\mathrm{C} 10$ & $1.501(4)$ & $\mathrm{C} 22-\mathrm{H} 22 \mathrm{~A}$ & 0.99 \\
\hline $\mathrm{C} 4-\mathrm{C} 5$ & $1.529(4)$ & $\mathrm{C} 22-\mathrm{H} 22 \mathrm{~B}$ & 0.99 \\
\hline $\mathrm{C} 4-\mathrm{H} 4 \mathrm{~A}$ & 1 & $\mathrm{C} 24-\mathrm{C} 29$ & $1.383(4)$ \\
\hline $\mathrm{C} 5-\mathrm{C} 6$ & $1.536(3)$ & $\mathrm{C} 24-\mathrm{C} 25$ & $1.394(4)$ \\
\hline $\mathrm{C} 5-\mathrm{H} 5 \mathrm{~A}$ & 0.99 & $\mathrm{C} 25-\mathrm{C} 26$ & $1.377(4)$ \\
\hline C5-H5B & 0.99 & $\mathrm{C} 25-\mathrm{H} 25 \mathrm{~A}$ & 0.95 \\
\hline $\mathrm{C} 6-\mathrm{C} 11$ & $1.511(3)$ & $\mathrm{C} 26-\mathrm{C} 27$ & $1.365(5)$ \\
\hline $\mathrm{C} 6-\mathrm{C} 9$ & $1.540(3)$ & $\mathrm{C} 26-\mathrm{H} 26 \mathrm{~A}$ & 0.95 \\
\hline $\mathrm{C} 7-\mathrm{C} 8$ & $1.528(4)$ & $\mathrm{C} 27-\mathrm{C} 28$ & $1.370(5)$ \\
\hline C7-H7A & 0.99 & $\mathrm{C} 27-\mathrm{H} 27 \mathrm{~A}$ & 0.95 \\
\hline $\mathrm{C} 7-\mathrm{H} 7 \mathrm{~B}$ & 0.99 & $\mathrm{C} 28-\mathrm{C} 29$ & $1.387(4)$ \\
\hline $\mathrm{C} 8-\mathrm{C} 10$ & $1.522(4)$ & $\mathrm{C} 28-\mathrm{H} 28 \mathrm{~A}$ & 0.95 \\
\hline $\mathrm{C} 8-\mathrm{C} 9$ & $1.539(4)$ & $\mathrm{C} 29-\mathrm{H} 29 \mathrm{~A}$ & 0.95 \\
\hline $\mathrm{C} 11-\mathrm{N} 1-\mathrm{C} 12$ & $108.39(19)$ & $\mathrm{N} 2-\mathrm{C} 11-\mathrm{N} 1$ & $110.2(2)$ \\
\hline $\mathrm{C} 11-\mathrm{N} 1-\mathrm{C} 13$ & $130.57(18)$ & $\mathrm{N} 2-\mathrm{C} 11-\mathrm{C} 6$ & $122.5(2)$ \\
\hline $\mathrm{C} 12-\mathrm{N} 1-\mathrm{C} 13$ & $121.01(19)$ & $\mathrm{N} 1-\mathrm{C} 11-\mathrm{C} 6$ & $127.21(19)$ \\
\hline $\mathrm{C} 11-\mathrm{N} 2-\mathrm{N} 3$ & $105.12(19)$ & $\mathrm{N} 3-\mathrm{C} 12-\mathrm{N} 1$ & $102.9(2)$ \\
\hline $\mathrm{C} 12-\mathrm{N} 3-\mathrm{N} 2$ & $113.44(18)$ & $\mathrm{N} 3-\mathrm{C} 12-\mathrm{S} 1$ & $130.23(18)$ \\
\hline $\mathrm{C} 12-\mathrm{N} 3-\mathrm{C} 19$ & $125.9(2)$ & $\mathrm{N} 1-\mathrm{C} 12-\mathrm{S} 1$ & $126.90(19)$ \\
\hline $\mathrm{N} 2-\mathrm{N} 3-\mathrm{C} 19$ & $120.1(2)$ & $\mathrm{C} 18-\mathrm{C} 13-\mathrm{C} 14$ & $120.9(2)$ \\
\hline $\mathrm{C} 19-\mathrm{N} 4-\mathrm{C} 20$ & $116.2(2)$ & $\mathrm{C} 18-\mathrm{C} 13-\mathrm{N} 1$ & $119.8(2)$ \\
\hline $\mathrm{C} 19-\mathrm{N} 4-\mathrm{C} 23$ & $114.0(2)$ & $\mathrm{C} 14-\mathrm{C} 13-\mathrm{N} 1$ & $119.3(2)$ \\
\hline $\mathrm{C} 20-\mathrm{N} 4-\mathrm{C} 23$ & $110.5(2)$ & $\mathrm{C} 15-\mathrm{C} 14-\mathrm{C} 13$ & $119.9(3)$ \\
\hline $\mathrm{C} 24-\mathrm{N} 5-\mathrm{C} 21$ & $116.6(2)$ & $\mathrm{C} 15-\mathrm{C} 14-\mathrm{H} 14 \mathrm{~A}$ & 120.1 \\
\hline $\mathrm{C} 24-\mathrm{N} 5-\mathrm{C} 22$ & $113.3(2)$ & $\mathrm{C} 13-\mathrm{C} 14-\mathrm{H} 14 \mathrm{~A}$ & 120.1 \\
\hline $\mathrm{C} 21-\mathrm{N} 5-\mathrm{C} 22$ & $109.8(2)$ & $\mathrm{C} 16-\mathrm{C} 15-\mathrm{C} 14$ & $118.1(3)$ \\
\hline $\mathrm{C} 2-\mathrm{C} 1-\mathrm{C} 6$ & $110.4(2)$ & $\mathrm{C} 16-\mathrm{C} 15-\mathrm{H} 15 \mathrm{~A}$ & 120.9 \\
\hline $\mathrm{C} 2-\mathrm{C} 1-\mathrm{H} 1 \mathrm{~A}$ & 109.6 & $\mathrm{C} 14-\mathrm{C} 15-\mathrm{H} 15 \mathrm{~A}$ & 120.9 \\
\hline
\end{tabular}




\begin{tabular}{|c|c|c|c|}
\hline $\mathrm{C} 6-\mathrm{C} 1-\mathrm{H} 1 \mathrm{~A}$ & 109.6 & $\mathrm{C} 15-\mathrm{C} 16-\mathrm{F} 1$ & $118.9(3)$ \\
\hline $\mathrm{C} 2-\mathrm{C} 1-\mathrm{H} 1 \mathrm{~B}$ & 109.6 & $\mathrm{C} 15-\mathrm{C} 16-\mathrm{C} 17$ & $123.8(3)$ \\
\hline $\mathrm{C} 6-\mathrm{C} 1-\mathrm{H} 1 \mathrm{~B}$ & 109.6 & $\mathrm{~F} 1-\mathrm{C} 16-\mathrm{C} 17$ & $117.3(3)$ \\
\hline $\mathrm{H} 1 \mathrm{~A}-\mathrm{C} 1-\mathrm{H} 1 \mathrm{~B}$ & 108.1 & $\mathrm{C} 16-\mathrm{C} 17-\mathrm{C} 18$ & $118.2(3)$ \\
\hline $\mathrm{C} 7-\mathrm{C} 2-\mathrm{C} 3$ & $109.6(3)$ & $\mathrm{C} 16-\mathrm{C} 17-\mathrm{H} 17 \mathrm{~A}$ & 120.9 \\
\hline $\mathrm{C} 7-\mathrm{C} 2-\mathrm{C} 1$ & $109.9(2)$ & $\mathrm{C} 18-\mathrm{C} 17-\mathrm{H} 17 \mathrm{~A}$ & 120.9 \\
\hline $\mathrm{C} 3-\mathrm{C} 2-\mathrm{C} 1$ & $109.2(2)$ & $\mathrm{C} 13-\mathrm{C} 18-\mathrm{C} 17$ & $119.1(3)$ \\
\hline $\mathrm{C} 7-\mathrm{C} 2-\mathrm{H} 2 \mathrm{~A}$ & 109.4 & $\mathrm{C} 13-\mathrm{C} 18-\mathrm{H} 18 \mathrm{~A}$ & 120.5 \\
\hline $\mathrm{C} 3-\mathrm{C} 2-\mathrm{H} 2 \mathrm{~A}$ & 109.4 & $\mathrm{C} 17-\mathrm{C} 18-\mathrm{H} 18 \mathrm{~A}$ & 120.5 \\
\hline $\mathrm{C} 1-\mathrm{C} 2-\mathrm{H} 2 \mathrm{~A}$ & 109.4 & $\mathrm{~N} 4-\mathrm{C} 19-\mathrm{N} 3$ & $116.3(2)$ \\
\hline $\mathrm{C} 4-\mathrm{C} 3-\mathrm{C} 2$ & $109.3(2)$ & $\mathrm{N} 4-\mathrm{C} 19-\mathrm{H} 19 \mathrm{~A}$ & 108.2 \\
\hline $\mathrm{C} 4-\mathrm{C} 3-\mathrm{H} 3 \mathrm{~A}$ & 109.8 & N3-C19-H19A & 108.2 \\
\hline $\mathrm{C} 2-\mathrm{C} 3-\mathrm{H} 3 \mathrm{~A}$ & 109.8 & $\mathrm{~N} 4-\mathrm{C} 19-\mathrm{H} 19 \mathrm{~B}$ & 108.2 \\
\hline $\mathrm{C} 4-\mathrm{C} 3-\mathrm{H} 3 \mathrm{~B}$ & 109.8 & N3-C19-H19B & 108.2 \\
\hline $\mathrm{C} 2-\mathrm{C} 3-\mathrm{H} 3 \mathrm{~B}$ & 109.8 & $\mathrm{H} 19 \mathrm{~A}-\mathrm{C} 19-\mathrm{H} 19 \mathrm{~B}$ & 107.4 \\
\hline $\mathrm{H} 3 \mathrm{~A}-\mathrm{C} 3-\mathrm{H} 3 \mathrm{~B}$ & 108.3 & $\mathrm{~N} 4-\mathrm{C} 20-\mathrm{C} 21$ & $109.7(2)$ \\
\hline $\mathrm{C} 10-\mathrm{C} 4-\mathrm{C} 3$ & $109.9(3)$ & $\mathrm{N} 4-\mathrm{C} 20-\mathrm{H} 20 \mathrm{~A}$ & 109.7 \\
\hline $\mathrm{C} 10-\mathrm{C} 4-\mathrm{C} 5$ & $109.9(2)$ & $\mathrm{C} 21-\mathrm{C} 20-\mathrm{H} 20 \mathrm{~A}$ & 109.7 \\
\hline $\mathrm{C} 3-\mathrm{C} 4-\mathrm{C} 5$ & $110.1(3)$ & $\mathrm{N} 4-\mathrm{C} 20-\mathrm{H} 20 \mathrm{~B}$ & 109.7 \\
\hline $\mathrm{C} 10-\mathrm{C} 4-\mathrm{H} 4 \mathrm{~A}$ & 109 & $\mathrm{C} 21-\mathrm{C} 20-\mathrm{H} 20 \mathrm{~B}$ & 109.7 \\
\hline $\mathrm{C} 3-\mathrm{C} 4-\mathrm{H} 4 \mathrm{~A}$ & 109 & $\mathrm{H} 20 \mathrm{~A}-\mathrm{C} 20-\mathrm{H} 20 \mathrm{~B}$ & 108.2 \\
\hline $\mathrm{C} 5-\mathrm{C} 4-\mathrm{H} 4 \mathrm{~A}$ & 109 & $\mathrm{~N} 5-\mathrm{C} 21-\mathrm{C} 20$ & $109.2(2)$ \\
\hline $\mathrm{C} 4-\mathrm{C} 5-\mathrm{C} 6$ & $110.0(2)$ & $\mathrm{N} 5-\mathrm{C} 21-\mathrm{H} 21 \mathrm{~A}$ & 109.8 \\
\hline $\mathrm{C} 4-\mathrm{C} 5-\mathrm{H} 5 \mathrm{~A}$ & 109.7 & $\mathrm{C} 20-\mathrm{C} 21-\mathrm{H} 21 \mathrm{~A}$ & 109.8 \\
\hline $\mathrm{C} 6-\mathrm{C} 5-\mathrm{H} 5 \mathrm{~A}$ & 109.7 & N5-C21-H21B & 109.8 \\
\hline $\mathrm{C} 4-\mathrm{C} 5-\mathrm{H} 5 \mathrm{~B}$ & 109.7 & $\mathrm{C} 20-\mathrm{C} 21-\mathrm{H} 21 \mathrm{~B}$ & 109.8 \\
\hline $\mathrm{C} 6-\mathrm{C} 5-\mathrm{H} 5 \mathrm{~B}$ & 109.7 & $\mathrm{H} 21 \mathrm{~A}-\mathrm{C} 21-\mathrm{H} 21 \mathrm{~B}$ & 108.3 \\
\hline $\mathrm{H} 5 \mathrm{~A}-\mathrm{C} 5-\mathrm{H} 5 \mathrm{~B}$ & 108.2 & $\mathrm{~N} 4-\mathrm{C} 23-\mathrm{C} 22$ & $109.7(2)$ \\
\hline $\mathrm{C} 11-\mathrm{C} 6-\mathrm{C} 5$ & $109.46(19)$ & $\mathrm{N} 4-\mathrm{C} 23-\mathrm{H} 23 \mathrm{~A}$ & 109.7 \\
\hline $\mathrm{C} 11-\mathrm{C} 6-\mathrm{C} 9$ & $113.41(18)$ & $\mathrm{C} 22-\mathrm{C} 23-\mathrm{H} 23 \mathrm{~A}$ & 109.7 \\
\hline $\mathrm{C} 5-\mathrm{C} 6-\mathrm{C} 9$ & $108.6(2)$ & $\mathrm{N} 4-\mathrm{C} 23-\mathrm{H} 23 \mathrm{~B}$ & 109.7 \\
\hline $\mathrm{C} 11-\mathrm{C} 6-\mathrm{C} 1$ & $108.72(18)$ & $\mathrm{C} 22-\mathrm{C} 23-\mathrm{H} 23 \mathrm{~B}$ & 109.7 \\
\hline $\mathrm{C} 5-\mathrm{C} 6-\mathrm{C} 1$ & $108.15(19)$ & $\mathrm{H} 23 \mathrm{~A}-\mathrm{C} 23-\mathrm{H} 23 \mathrm{~B}$ & 108.2 \\
\hline $\mathrm{C} 9-\mathrm{C} 6-\mathrm{C} 1$ & $108.4(2)$ & $\mathrm{N} 5-\mathrm{C} 22-\mathrm{C} 23$ & $110.3(2)$ \\
\hline $\mathrm{C} 2-\mathrm{C} 7-\mathrm{C} 8$ & $108.9(2)$ & $\mathrm{N} 5-\mathrm{C} 22-\mathrm{H} 22 \mathrm{~A}$ & 109.6 \\
\hline $\mathrm{C} 2-\mathrm{C} 7-\mathrm{H} 7 \mathrm{~A}$ & 109.9 & $\mathrm{C} 23-\mathrm{C} 22-\mathrm{H} 22 \mathrm{~A}$ & 109.6 \\
\hline $\mathrm{C} 8-\mathrm{C} 7-\mathrm{H} 7 \mathrm{~A}$ & 109.9 & $\mathrm{~N} 5-\mathrm{C} 22-\mathrm{H} 22 \mathrm{~B}$ & 109.6 \\
\hline $\mathrm{C} 2-\mathrm{C} 7-\mathrm{H} 7 \mathrm{~B}$ & 109.9 & $\mathrm{C} 23-\mathrm{C} 22-\mathrm{H} 22 \mathrm{~B}$ & 109.6 \\
\hline $\mathrm{C} 8-\mathrm{C} 7-\mathrm{H} 7 \mathrm{~B}$ & 109.9 & $\mathrm{H} 22 \mathrm{~A}-\mathrm{C} 22-\mathrm{H} 22 \mathrm{~B}$ & 108.1 \\
\hline $\mathrm{H} 7 \mathrm{~A}-\mathrm{C} 7-\mathrm{H} 7 \mathrm{~B}$ & 108.3 & $\mathrm{C} 29-\mathrm{C} 24-\mathrm{C} 25$ & $118.1(3)$ \\
\hline $\mathrm{C} 10-\mathrm{C} 8-\mathrm{C} 7$ & $110.3(2)$ & $\mathrm{C} 29-\mathrm{C} 24-\mathrm{N} 5$ & $123.6(2)$ \\
\hline $\mathrm{C} 10-\mathrm{C} 8-\mathrm{C} 9$ & $109.7(2)$ & $\mathrm{C} 25-\mathrm{C} 24-\mathrm{N} 5$ & $118.3(2)$ \\
\hline $\mathrm{C} 7-\mathrm{C} 8-\mathrm{C} 9$ & $109.1(2)$ & $\mathrm{C} 26-\mathrm{C} 25-\mathrm{C} 24$ & $120.4(3)$ \\
\hline $\mathrm{C} 10-\mathrm{C} 8-\mathrm{H} 8 \mathrm{~A}$ & 109.2 & $\mathrm{C} 26-\mathrm{C} 25-\mathrm{H} 25 \mathrm{~A}$ & 119.8 \\
\hline $\mathrm{C} 7-\mathrm{C} 8-\mathrm{H} 8 \mathrm{~A}$ & 109.2 & $\mathrm{C} 24-\mathrm{C} 25-\mathrm{H} 25 \mathrm{~A}$ & 119.8 \\
\hline $\mathrm{C} 9-\mathrm{C} 8-\mathrm{H} 8 \mathrm{~A}$ & 109.2 & $\mathrm{C} 27-\mathrm{C} 26-\mathrm{C} 25$ & $120.8(3)$ \\
\hline $\mathrm{C} 8-\mathrm{C} 9-\mathrm{C} 6$ & $109.8(2)$ & $\mathrm{C} 27-\mathrm{C} 26-\mathrm{H} 26 \mathrm{~A}$ & 119.6 \\
\hline $\mathrm{C} 8-\mathrm{C} 9-\mathrm{H} 9 \mathrm{~A}$ & 109.7 & $\mathrm{C} 25-\mathrm{C} 26-\mathrm{H} 26 \mathrm{~A}$ & 119.6 \\
\hline
\end{tabular}




\begin{tabular}{|c|c|c|c|}
\hline $\mathrm{C} 6-\mathrm{C} 9-\mathrm{H} 9 \mathrm{~A}$ & 109.7 & $\mathrm{C} 26-\mathrm{C} 27-\mathrm{C} 28$ & $119.8(3)$ \\
\hline $\mathrm{C} 8-\mathrm{C} 9-\mathrm{H} 9 \mathrm{~B}$ & 109.7 & $\mathrm{C} 26-\mathrm{C} 27-\mathrm{H} 27 \mathrm{~A}$ & 120.1 \\
\hline C6- $69-\mathrm{H} 9 \mathrm{~B}$ & 109.7 & $\mathrm{C} 28-\mathrm{C} 27-\mathrm{H} 27 \mathrm{~A}$ & 120.1 \\
\hline $\mathrm{H} 9 \mathrm{~A}-\mathrm{C} 9-\mathrm{H} 9 \mathrm{~B}$ & 108.2 & $\mathrm{C} 27-\mathrm{C} 28-\mathrm{C} 29$ & $120.1(3)$ \\
\hline $\mathrm{C} 4-\mathrm{C} 10-\mathrm{C} 8$ & $109.3(2)$ & $\mathrm{C} 27-\mathrm{C} 28-\mathrm{H} 28 \mathrm{~A}$ & 120 \\
\hline $\mathrm{C} 4-\mathrm{C} 10-\mathrm{H} 10 \mathrm{~A}$ & 109.8 & $\mathrm{C} 29-\mathrm{C} 28-\mathrm{H} 28 \mathrm{~A}$ & 120 \\
\hline $\mathrm{C} 8-\mathrm{C} 10-\mathrm{H} 10 \mathrm{~A}$ & 109.8 & $\mathrm{C} 24-\mathrm{C} 29-\mathrm{C} 28$ & $120.8(3)$ \\
\hline $\mathrm{C} 4-\mathrm{C} 10-\mathrm{H} 10 \mathrm{~B}$ & 109.8 & $\mathrm{C} 24-\mathrm{C} 29-\mathrm{H} 29 \mathrm{~A}$ & 119.6 \\
\hline $\mathrm{C} 8-\mathrm{C} 10-\mathrm{H} 10 \mathrm{~B}$ & 109.8 & $\mathrm{C} 28-\mathrm{C} 29-\mathrm{H} 29 \mathrm{~A}$ & 119.6 \\
\hline $\mathrm{H} 10 \mathrm{~A}-\mathrm{C} 10-\mathrm{H} 10 \mathrm{~B}$ & 108.3 & & \\
\hline $\mathrm{C} 11-\mathrm{N} 2-\mathrm{N} 3-\mathrm{C} 12$ & $-0.5(3)$ & $\mathrm{C} 11-\mathrm{N} 1-\mathrm{C} 12-\mathrm{N} 3$ & $-0.6(2)$ \\
\hline $\mathrm{C} 11-\mathrm{N} 2-\mathrm{N} 3-\mathrm{C} 19$ & $-172.6(2)$ & $\mathrm{C} 13-\mathrm{N} 1-\mathrm{C} 12-\mathrm{N} 3$ & $-178.6(2)$ \\
\hline $\mathrm{C} 6-\mathrm{C} 1-\mathrm{C} 2-\mathrm{C} 7$ & $-59.7(3)$ & $\mathrm{C} 11-\mathrm{N} 1-\mathrm{C} 12-\mathrm{S} 1$ & $178.75(18)$ \\
\hline $\mathrm{C} 6-\mathrm{C} 1-\mathrm{C} 2-\mathrm{C} 3$ & $60.6(3)$ & $\mathrm{C} 13-\mathrm{N} 1-\mathrm{C} 12-\mathrm{S} 1$ & $0.8(3)$ \\
\hline $\mathrm{C} 7-\mathrm{C} 2-\mathrm{C} 3-\mathrm{C} 4$ & $60.3(3)$ & $\mathrm{C} 11-\mathrm{N} 1-\mathrm{C} 13-\mathrm{C} 18$ & $-83.5(3)$ \\
\hline $\mathrm{C} 1-\mathrm{C} 2-\mathrm{C} 3-\mathrm{C} 4$ & $-60.2(3)$ & $\mathrm{C} 12-\mathrm{N} 1-\mathrm{C} 13-\mathrm{C} 18$ & $94.0(3)$ \\
\hline $\mathrm{C} 2-\mathrm{C} 3-\mathrm{C} 4-\mathrm{C} 10$ & $-60.7(3)$ & $\mathrm{C} 11-\mathrm{N} 1-\mathrm{C} 13-\mathrm{C} 14$ & $99.5(3)$ \\
\hline $\mathrm{C} 2-\mathrm{C} 3-\mathrm{C} 4-\mathrm{C} 5$ & $60.4(3)$ & $\mathrm{C} 12-\mathrm{N} 1-\mathrm{C} 13-\mathrm{C} 14$ & $-83.1(3)$ \\
\hline $\mathrm{C} 10-\mathrm{C} 4-\mathrm{C} 5-\mathrm{C} 6$ & $60.9(3)$ & $\mathrm{C} 18-\mathrm{C} 13-\mathrm{C} 14-\mathrm{C} 15$ & $2.3(4)$ \\
\hline $\mathrm{C} 3-\mathrm{C} 4-\mathrm{C} 5-\mathrm{C} 6$ & $-60.2(3)$ & $\mathrm{N} 1-\mathrm{C} 13-\mathrm{C} 14-\mathrm{C} 15$ & $179.3(2)$ \\
\hline $\mathrm{C} 4-\mathrm{C} 5-\mathrm{C} 6-\mathrm{C} 11$ & $177.0(2)$ & $\mathrm{C} 13-\mathrm{C} 14-\mathrm{C} 15-\mathrm{C} 16$ & $-0.6(4)$ \\
\hline $\mathrm{C} 4-\mathrm{C} 5-\mathrm{C} 6-\mathrm{C} 9$ & $-58.7(3)$ & $\mathrm{C} 14-\mathrm{C} 15-\mathrm{C} 16-\mathrm{F} 1$ & $-179.9(3)$ \\
\hline $\mathrm{C} 4-\mathrm{C} 5-\mathrm{C} 6-\mathrm{C} 1$ & $58.7(3)$ & $\mathrm{C} 14-\mathrm{C} 15-\mathrm{C} 16-\mathrm{C} 17$ & $-0.8(5)$ \\
\hline $\mathrm{C} 2-\mathrm{C} 1-\mathrm{C} 6-\mathrm{C} 11$ & $-178.1(2)$ & $\mathrm{C} 15-\mathrm{C} 16-\mathrm{C} 17-\mathrm{C} 18$ & $0.5(5)$ \\
\hline $\mathrm{C} 2-\mathrm{C} 1-\mathrm{C} 6-\mathrm{C} 5$ & $-59.4(3)$ & $\mathrm{F} 1-\mathrm{C} 16-\mathrm{C} 17-\mathrm{C} 18$ & $179.6(2)$ \\
\hline $\mathrm{C} 2-\mathrm{C} 1-\mathrm{C} 6-\mathrm{C} 9$ & $58.2(3)$ & $\mathrm{C} 14-\mathrm{C} 13-\mathrm{C} 18-\mathrm{C} 17$ & $-2.5(4)$ \\
\hline $\mathrm{C} 3-\mathrm{C} 2-\mathrm{C} 7-\mathrm{C} 8$ & $-59.2(3)$ & $\mathrm{N} 1-\mathrm{C} 13-\mathrm{C} 18-\mathrm{C} 17$ & $-179.5(2)$ \\
\hline $\mathrm{C} 1-\mathrm{C} 2-\mathrm{C} 7-\mathrm{C} 8$ & $60.8(3)$ & $\mathrm{C} 16-\mathrm{C} 17-\mathrm{C} 18-\mathrm{C} 13$ & $1.2(4)$ \\
\hline $\mathrm{C} 2-\mathrm{C} 7-\mathrm{C} 8-\mathrm{C} 10$ & $59.0(3)$ & $\mathrm{C} 20-\mathrm{N} 4-\mathrm{C} 19-\mathrm{N} 3$ & $69.5(3)$ \\
\hline $\mathrm{C} 2-\mathrm{C} 7-\mathrm{C} 8-\mathrm{C} 9$ & $-61.5(3)$ & $\mathrm{C} 23-\mathrm{N} 4-\mathrm{C} 19-\mathrm{N} 3$ & $-60.7(3)$ \\
\hline $\mathrm{C} 10-\mathrm{C} 8-\mathrm{C} 9-\mathrm{C} 6$ & $-59.5(3)$ & $\mathrm{C} 12-\mathrm{N} 3-\mathrm{C} 19-\mathrm{N} 4$ & $102.6(3)$ \\
\hline $\mathrm{C} 7-\mathrm{C} 8-\mathrm{C} 9-\mathrm{C} 6$ & $61.4(3)$ & $\mathrm{N} 2-\mathrm{N} 3-\mathrm{C} 19-\mathrm{N} 4$ & $-86.3(3)$ \\
\hline $\mathrm{C} 11-\mathrm{C} 6-\mathrm{C} 9-\mathrm{C} 8$ & $-179.9(2)$ & $\mathrm{C} 19-\mathrm{N} 4-\mathrm{C} 20-\mathrm{C} 21$ & $168.6(2)$ \\
\hline $\mathrm{C} 5-\mathrm{C} 6-\mathrm{C} 9-\mathrm{C} 8$ & $58.2(3)$ & $\mathrm{C} 23-\mathrm{N} 4-\mathrm{C} 20-\mathrm{C} 21$ & $-59.5(3)$ \\
\hline $\mathrm{C} 1-\mathrm{C} 6-\mathrm{C} 9-\mathrm{C} 8$ & $-59.1(3)$ & $\mathrm{C} 24-\mathrm{N} 5-\mathrm{C} 21-\mathrm{C} 20$ & $169.9(2)$ \\
\hline $\mathrm{C} 3-\mathrm{C} 4-\mathrm{C} 10-\mathrm{C} 8$ & $60.1(3)$ & $\mathrm{C} 22-\mathrm{N} 5-\mathrm{C} 21-\mathrm{C} 20$ & $-59.5(3)$ \\
\hline $\mathrm{C} 5-\mathrm{C} 4-\mathrm{C} 10-\mathrm{C} 8$ & $-61.2(3)$ & $\mathrm{N} 4-\mathrm{C} 20-\mathrm{C} 21-\mathrm{N} 5$ & $59.9(3)$ \\
\hline $\mathrm{C} 7-\mathrm{C} 8-\mathrm{C} 10-\mathrm{C} 4$ & $-59.6(3)$ & $\mathrm{C} 19-\mathrm{N} 4-\mathrm{C} 23-\mathrm{C} 22$ & $-168.7(2)$ \\
\hline $\mathrm{C} 9-\mathrm{C} 8-\mathrm{C} 10-\mathrm{C} 4$ & $60.6(3)$ & $\mathrm{C} 20-\mathrm{N} 4-\mathrm{C} 23-\mathrm{C} 22$ & $58.3(3)$ \\
\hline $\mathrm{N} 3-\mathrm{N} 2-\mathrm{C} 11-\mathrm{N} 1$ & $0.1(2)$ & $\mathrm{C} 24-\mathrm{N} 5-\mathrm{C} 22-\mathrm{C} 23$ & $-168.7(2)$ \\
\hline $\mathrm{N} 3-\mathrm{N} 2-\mathrm{C} 11-\mathrm{C} 6$ & $176.33(19)$ & $\mathrm{C} 21-\mathrm{N} 5-\mathrm{C} 22-\mathrm{C} 23$ & $59.0(3)$ \\
\hline $\mathrm{C} 12-\mathrm{N} 1-\mathrm{C} 11-\mathrm{N} 2$ & $0.3(3)$ & $\mathrm{N} 4-\mathrm{C} 23-\mathrm{C} 22-\mathrm{N} 5$ & $-57.8(3)$ \\
\hline $\mathrm{C} 13-\mathrm{N} 1-\mathrm{C} 11-\mathrm{N} 2$ & $178.0(2)$ & $\mathrm{C} 21-\mathrm{N} 5-\mathrm{C} 24-\mathrm{C} 29$ & $15.0(4)$ \\
\hline $\mathrm{C} 12-\mathrm{N} 1-\mathrm{C} 11-\mathrm{C} 6$ & $-175.7(2)$ & $\mathrm{C} 22-\mathrm{N} 5-\mathrm{C} 24-\mathrm{C} 29$ & $-113.9(3)$ \\
\hline $\mathrm{C} 13-\mathrm{N} 1-\mathrm{C} 11-\mathrm{C} 6$ & $2.0(4)$ & $\mathrm{C} 21-\mathrm{N} 5-\mathrm{C} 24-\mathrm{C} 25$ & $-165.1(3)$ \\
\hline $\mathrm{C} 5-\mathrm{C} 6-\mathrm{C} 11-\mathrm{N} 2$ & $-97.1(3)$ & $\mathrm{C} 22-\mathrm{N} 5-\mathrm{C} 24-\mathrm{C} 25$ & $66.0(3)$ \\
\hline $\mathrm{C} 9-\mathrm{C} 6-\mathrm{C} 11-\mathrm{N} 2$ & $141.4(2)$ & $\mathrm{C} 29-\mathrm{C} 24-\mathrm{C} 25-\mathrm{C} 26$ & $0.5(4)$ \\
\hline
\end{tabular}




$\begin{array}{llll}\mathrm{C} 1-\mathrm{C} 6-\mathrm{C} 11-\mathrm{N} 2 & 20.8(3) & \mathrm{N} 5-\mathrm{C} 24-\mathrm{C} 25-\mathrm{C} 26 & -179.4(3) \\ \mathrm{C} 5-\mathrm{C} 6-\mathrm{C} 11-\mathrm{N} 1 & 78.4(3) & \mathrm{C} 24-\mathrm{C} 25-\mathrm{C} 26-\mathrm{C} 27 & 0.4(5) \\ \mathrm{C} 9-\mathrm{C} 6-\mathrm{C} 11-\mathrm{N} 1 & -43.0(3) & \mathrm{C} 25-\mathrm{C} 26-\mathrm{C} 27-\mathrm{C} 28 & -1.0(5) \\ \mathrm{C} 1-\mathrm{C} 6-\mathrm{C} 11-\mathrm{N} 1 & -163.6(2) & \mathrm{C} 26-\mathrm{C} 27-\mathrm{C} 28-\mathrm{C} 29 & 0.7(5) \\ \mathrm{N} 2-\mathrm{N} 3-\mathrm{C} 12-\mathrm{N} 1 & 0.7(3) & \mathrm{C} 25-\mathrm{C} 24-\mathrm{C} 29-\mathrm{C} 28 & -0.8(4) \\ \mathrm{C} 19-\mathrm{N} 3-\mathrm{C} 12-\mathrm{N} 1 & 172.3(2) & \mathrm{N} 5-\mathrm{C} 24-\mathrm{C} 29-\mathrm{C} 28 & 179.1(3) \\ \mathrm{N} 2-\mathrm{N} 3-\mathrm{C} 12-\mathrm{S} 1 & -178.63(18) & \mathrm{C} 27-\mathrm{C} 28-\mathrm{C} 29-\mathrm{C} 24 & 0.2(5) \\ \mathrm{C} 19-\mathrm{N} 3-\mathrm{C} 12-\mathrm{S} 1 & -7.1(4) & & \end{array}$

Hydrogen-bond geometry $\left(\AA,{ }^{\circ}\right)$

\begin{tabular}{lllll}
\hline$D-\mathrm{H} \cdots A$ & $D-\mathrm{H}$ & $\mathrm{H} \cdots A$ & $D \cdots A$ & $D-\mathrm{H} \cdots A$ \\
\hline $\mathrm{C} 22-\mathrm{H} 22 B \cdots \mathrm{F} 1^{\mathrm{i}}$ & 0.99 & 2.49 & $3.332(4)$ & 142 \\
\hline
\end{tabular}

Symmetry code: (i) $-x+2,-y+1,-z+1$.

3-(Adamantan-1-yl)-4-(4-fluorophenyl)-1-\{[4-(2-methoxyphenyl)piperazin-1-yl]methyl\}-4,5-dihydro-1 H-1,2,4-

triazole-5-thione (II)

Crystal data

$\mathrm{C}_{30} \mathrm{H}_{36} \mathrm{FN}_{5} \mathrm{OS}$

$M_{r}=533.7$

Monoclinic, $P 2_{1} / c$

Hall symbol: -P 2ybc

$a=11.3074$ (7) $\AA$

$b=12.1576(8) \AA$

$c=20.4976(13) \AA$

$\beta=101.328(2)^{\circ}$

$V=2762.9(3) \AA^{3}$

$Z=4$

\section{Data collection}

\section{Bruker APEXII CCD}

diffractometer

Radiation source: fine-focus sealed tube

Graphite monochromator

$\varphi$ and $\omega$ scans

Absorption correction: multi-scan

(SADABS; Bruker, 2012)

$T_{\min }=0.965, T_{\max }=0.975$

\section{Refinement}

Refinement on $F^{2}$

Least-squares matrix: full

$R\left[F^{2}>2 \sigma\left(F^{2}\right)\right]=0.055$

$w R\left(F^{2}\right)=0.133$

$S=1.01$

6347 reflections

344 parameters

0 restraints

0 constraints

Primary atom site location: structure-invariant direct methods
$F(000)=1136$

$D_{\mathrm{x}}=1.283 \mathrm{Mg} \mathrm{m}^{-3}$

Mo $K \alpha$ radiation, $\lambda=0.71073 \AA$

Cell parameters from 8790 reflections

$\theta=2.5-22.8^{\circ}$

$\mu=0.16 \mathrm{~mm}^{-1}$

$T=293 \mathrm{~K}$

Block, colourless

$0.42 \times 0.19 \times 0.16 \mathrm{~mm}$

67287 measured reflections

6350 independent reflections

3203 reflections with $I>2 \sigma(I)$

$R_{\text {int }}=0.134$

$\theta_{\text {max }}=27.5^{\circ}, \theta_{\min }=2.5^{\circ}$

$h=-14 \rightarrow 14$

$k=-15 \rightarrow 15$

$l=-26 \rightarrow 26$

Secondary atom site location: difference Fourier map

Hydrogen site location: inferred from neighbouring sites

$\mathrm{H}$-atom parameters constrained

$w=1 /\left[\sigma^{2}\left(F_{\mathrm{o}}^{2}\right)+(0.0452 P)^{2}+0.8222 P\right]$

where $P=\left(F_{\mathrm{o}}{ }^{2}+2 F_{\mathrm{c}}{ }^{2}\right) / 3$

$(\Delta / \sigma)_{\max }<0.001$

$\Delta \rho_{\max }=0.16 \mathrm{e} \AA^{-3}$

$\Delta \rho_{\min }=-0.22 \mathrm{e}^{-3}$ 


\section{Special details}

Geometry. All esds (except the esd in the dihedral angle between two 1.s. planes) are estimated using the full covariance matrix. The cell esds are taken into account individually in the estimation of esds in distances, angles and torsion angles; correlations between esds in cell parameters are only used when they are defined by crystal symmetry. An approximate (isotropic) treatment of cell esds is used for estimating esds involving l.s. planes.

Refinement. Refinement of $\mathrm{F}^{2}$ against ALL reflections. The weighted R-factor $\mathrm{wR}$ and goodness of fit $\mathrm{S}$ are based on $\mathrm{F}^{2}$, conventional $R$-factors $R$ are based on $F$, with $F$ set to zero for negative $F^{2}$. The threshold expression of $F^{2}>2 \operatorname{sigma}\left(\mathrm{F}^{2}\right)$ is used only for calculating R-factors(gt) etc. and is not relevant to the choice of reflections for refinement. R-factors based on $\mathrm{F}^{2}$ are statistically about twice as large as those based on F, and R- factors based on ALL data will be even larger.

Fractional atomic coordinates and isotropic or equivalent isotropic displacement parameters $\left(\hat{A}^{2}\right)$

\begin{tabular}{|c|c|c|c|c|}
\hline & $x$ & $y$ & $z$ & $U_{\text {iso }} * / U_{\text {eq }}$ \\
\hline S1 & $0.24167(6)$ & $0.32667(6)$ & $0.43345(3)$ & $0.0608(2)$ \\
\hline $\mathrm{F} 1$ & $0.3856(2)$ & $0.79567(15)$ & $0.45842(10)$ & $0.1199(8)$ \\
\hline $\mathrm{O} 1$ & $0.34615(16)$ & $-0.06806(14)$ & $0.77358(8)$ & $0.0618(5)$ \\
\hline N1 & $0.19559(16)$ & $0.42142(14)$ & $0.54744(10)$ & $0.0428(5)$ \\
\hline N3 & $0.13241(17)$ & $0.25598(15)$ & $0.53226(9)$ & $0.0469(5)$ \\
\hline $\mathrm{N} 2$ & $0.10390(17)$ & $0.28893(15)$ & $0.59193(10)$ & $0.0481(5)$ \\
\hline N4 & $0.18293(18)$ & $0.05911(15)$ & $0.54290(9)$ & $0.0476(5)$ \\
\hline N5 & $0.35533(17)$ & $-0.04464(14)$ & $0.64472(9)$ & $0.0439(5)$ \\
\hline $\mathrm{C} 1$ & $0.0665(2)$ & $0.5658(2)$ & $0.64261(13)$ & $0.0583(7)$ \\
\hline H1A & 0.1087 & 0.6099 & 0.615 & $0.07 *$ \\
\hline $\mathrm{H} 1 \mathrm{~B}$ & -0.0137 & 0.5502 & 0.6174 & $0.07^{*}$ \\
\hline $\mathrm{C} 2$ & $0.0572(3)$ & $0.6293(2)$ & $0.70571(14)$ & $0.0645(8)$ \\
\hline $\mathrm{H} 2 \mathrm{~A}$ & 0.014 & 0.6984 & 0.6932 & $0.077 *$ \\
\hline $\mathrm{C} 3$ & $-0.0100(3)$ & $0.5635(2)$ & $0.74851(16)$ & $0.0755(9)$ \\
\hline $\mathrm{H} 3 \mathrm{~A}$ & -0.0907 & 0.5476 & 0.7242 & $0.091 *$ \\
\hline $\mathrm{H} 3 \mathrm{~B}$ & -0.0167 & 0.6053 & 0.7879 & $0.091 *$ \\
\hline $\mathrm{C} 4$ & $0.0574(3)$ & $0.4566(2)$ & $0.76852(15)$ & $0.0750(9)$ \\
\hline $\mathrm{H} 4 \mathrm{~A}$ & 0.0135 & 0.4131 & 0.7962 & $0.09 *$ \\
\hline $\mathrm{C} 5$ & $0.0666(3)$ & $0.3909(2)$ & $0.70539(14)$ & $0.0680(8)$ \\
\hline $\mathrm{H} 5 \mathrm{~A}$ & 0.1084 & 0.3221 & 0.7179 & $0.082 *$ \\
\hline $\mathrm{H} 5 \mathrm{~B}$ & -0.0137 & 0.3739 & 0.6808 & $0.082 *$ \\
\hline $\mathrm{C} 6$ & $0.13488(19)$ & $0.45742(17)$ & $0.66124(11)$ & $0.0412(6)$ \\
\hline $\mathrm{C} 7$ & $0.1822(3)$ & $0.6551(2)$ & $0.74427(15)$ & $0.0741(9)$ \\
\hline $\mathrm{H} 7 \mathrm{~A}$ & 0.2254 & 0.6987 & 0.717 & $0.089 *$ \\
\hline $\mathrm{H} 7 \mathrm{~B}$ & 0.1765 & 0.6975 & 0.7836 & $0.089^{*}$ \\
\hline $\mathrm{C} 8$ & $0.2498(2)$ & $0.5495(2)$ & $0.76445(14)$ & $0.0688(8)$ \\
\hline H8A & 0.3305 & 0.5668 & 0.7897 & $0.083^{*}$ \\
\hline C9 & $0.2610(2)$ & $0.4844(2)$ & $0.70174(13)$ & $0.0596(7)$ \\
\hline H9A & 0.3051 & 0.4168 & 0.7143 & $0.072 *$ \\
\hline H9B & 0.3054 & 0.5274 & 0.6748 & $0.072 *$ \\
\hline $\mathrm{C} 10$ & $0.1828(3)$ & $0.4810(3)$ & $0.80713(14)$ & $0.0807(9)$ \\
\hline H10A & 0.2258 & 0.4127 & 0.8194 & $0.097 *$ \\
\hline H10B & 0.1779 & 0.5207 & 0.8476 & $0.097^{*}$ \\
\hline C11 & $0.14343(19)$ & $0.39012(18)$ & 0.60057 (11) & $0.0413(6)$ \\
\hline $\mathrm{C} 12$ & $0.1899(2)$ & $0.33355(19)$ & $0.50378(12)$ & $0.0455(6)$ \\
\hline
\end{tabular}




$\begin{array}{lllll}\text { C13 } & 0.2440(2) & 0.52480(18) & 0.53089(11) & 0.0429(6) \\ \text { C14 } & 0.3666(2) & 0.5415(2) & 0.54450(13) & 0.0610(7) \\ \text { H14A } & 0.4173 & 0.4902 & 0.5695 & 0.073^{*} \\ \text { C15 } & 0.4142(3) & 0.6341(3) & 0.52107(16) & 0.0785(10) \\ \text { H15A } & 0.4969 & 0.6466 & 0.53 & 0.094^{*} \\ \text { C16 } & 0.3373(4) & 0.7071(2) & 0.48460(16) & 0.0740(9) \\ \text { C17 } & 0.2163(3) & 0.6944(2) & 0.47141(14) & 0.0666(8) \\ \text { H17A } & 0.1662 & 0.7472 & 0.4474 & 0.08^{*} \\ \text { C18 } & 0.1692(2) & 0.60109(19) & 0.49462(12) & 0.0514(6) \\ \text { H18A } & 0.0862 & 0.5897 & 0.4857 & 0.062^{*} \\ \text { C19 } & 0.1079(2) & 0.14220(19) & 0.50729(12) & 0.0550(7) \\ \text { H19A } & 0.0246 & 0.1246 & 0.5083 & 0.066^{*} \\ \text { H19B } & 0.1165 & 0.1399 & 0.4612 & 0.066^{*} \\ \text { C20 } & 0.3118(2) & 0.0819(2) & 0.55064(12) & 0.0541(7) \\ \text { H20A } & 0.3322 & 0.146 & 0.5788 & 0.065^{*} \\ \text { H20B } & 0.3305 & 0.0983 & 0.5075 & 0.065^{*} \\ \text { C21 } & 0.3869(2) & -0.0149(2) & 0.58108(12) & 0.0560(7) \\ \text { H21A } & 0.3728 & -0.0772 & 0.551 & 0.067^{*} \\ \text { H21B } & 0.4719 & 0.0039 & 0.588 & 0.067^{*} \\ \text { C22 } & 0.2259(2) & -0.06913(19) & 0.63489(12) & 0.0505(6) \\ \text { H22A } & 0.2053 & -0.0894 & 0.677 & 0.061^{*} \\ \text { H22B } & 0.2066 & -0.1307 & 0.6046 & 0.061^{*} \\ \text { C23 } & 0.1536(2) & 0.03040(19) & 0.60675(12) & 0.0483(6) \\ \text { H23A } & 0.0681 & 0.0145 & 0.6011 & 0.058^{*} \\ \text { H23B } & 0.1721 & 0.0918 & 0.6373 & 0.058^{*} \\ \text { C24 } & 0.4291(2) & -0.12587(18) & 0.68294(12) & 0.0453(6) \\ \text { C25 } & 0.5089(2) & -0.1917(2) & 0.65777(13) & 0.0592(7) \\ \text { H25A } & 0.5185 & -0.1814 & 0.6142 & 0.071^{*} \\ \text { C26 } & 0.5751(3) & -0.2730(2) & 0.69618(16) & 0.0722(8) \\ \text { H26A } & 0.6289 & -0.316 & 0.6783 & 0.087^{*} \\ \text { C27 } & 0.5616(2) & -0.2903(2) & 0.76022(16) & 0.0686(8) \\ \text { H27A } & 0.6031 & -0.3471 & 0.7852 & 0.082^{*} \\ \text { C28 } & 0.4857(2) & -0.2226(2) & 0.78764(13) & 0.0591(7) \\ \text { H28A } & 0.4781 & -0.2326 & 0.8316 & 0.071^{*} \\ \text { C29 } & 0.4211(2) & -0.14002(19) & 0.74968(13) & 0.0482(6) \\ \text { C30 } & 0.3295(3) & -0.0813(2) & 0.83902(13) & 0.0715(8) \\ \text { H30A } & 0.2762 & -0.0251 & 0.8491 & 0.107^{*} \\ \text { H30B } & 0.4059 & -0.0755 & 0.8691 & 0.107^{*} \\ \text { H30C } & 0.295 & -0.1523 & 0.8437 & 0.107^{*}\end{array}$

Atomic displacement parameters $\left(\AA^{2}\right)$

\begin{tabular}{lllllll}
\hline & $U^{11}$ & $U^{22}$ & $U^{33}$ & $U^{12}$ & $U^{13}$ & $U^{23}$ \\
\hline S1 & $0.0707(5)$ & $0.0622(4)$ & $0.0515(4)$ & $0.0101(4)$ & $0.0172(3)$ & $-0.0018(3)$ \\
F1 & $0.190(2)$ & $0.0819(13)$ & $0.1131(15)$ & $-0.0717(13)$ & $0.0917(15)$ & $-0.0292(11)$ \\
O1 & $0.0783(13)$ & $0.0624(12)$ & $0.0492(11)$ & $0.0115(10)$ & $0.0236(9)$ & $0.0040(9)$ \\
N1 & $0.0404(11)$ & $0.0354(11)$ & $0.0527(12)$ & $0.0050(9)$ & $0.0089(9)$ & $-0.0016(10)$ \\
N3 & $0.0552(12)$ & $0.0383(11)$ & $0.0467(12)$ & $0.0066(10)$ & $0.0083(10)$ & $-0.0046(10)$
\end{tabular}




\begin{tabular}{|c|c|c|c|c|c|c|}
\hline N2 & $0.0510(12)$ & $0.0386(12)$ & $0.0548(13)$ & $0.0025(9)$ & $0.0107(10)$ & $-0.0056(10)$ \\
\hline N4 & $0.0623(14)$ & $0.0379(11)$ & $0.0429(12)$ & $0.0048(10)$ & $0.0111(10)$ & $-0.0022(9)$ \\
\hline N5 & $0.0504(12)$ & $0.0391(11)$ & $0.0444(12)$ & $0.0031(9)$ & $0.0144(9)$ & $0.0027(9)$ \\
\hline $\mathrm{C} 1$ & $0.0616(17)$ & $0.0520(16)$ & $0.0596(17)$ & $0.0177(13)$ & $0.0078(13)$ & $-0.0084(13)$ \\
\hline $\mathrm{C} 2$ & $0.078(2)$ & $0.0475(15)$ & $0.0713(19)$ & $0.0150(14)$ & $0.0220(17)$ & $-0.0104(14)$ \\
\hline $\mathrm{C} 3$ & $0.0684(19)$ & $0.074(2)$ & $0.093(2)$ & $-0.0041(16)$ & $0.0361(17)$ & $-0.0302(17)$ \\
\hline $\mathrm{C} 4$ & $0.104(2)$ & $0.0573(18)$ & $0.080(2)$ & $-0.0273(17)$ & $0.059(2)$ & $-0.0141(16)$ \\
\hline $\mathrm{C} 5$ & $0.087(2)$ & $0.0501(16)$ & 0.077 (2) & $-0.0167(14)$ & $0.0416(17)$ & $-0.0140(14)$ \\
\hline $\mathrm{C} 6$ & $0.0379(13)$ & $0.0357(13)$ & $0.0507(14)$ & $0.0002(10)$ & $0.0109(11)$ & $-0.0047(11)$ \\
\hline $\mathrm{C} 7$ & $0.100(2)$ & $0.0554(18)$ & $0.073(2)$ & $-0.0244(17)$ & $0.0324(18)$ & $-0.0197(15)$ \\
\hline $\mathrm{C} 8$ & $0.0542(17)$ & $0.088(2)$ & $0.0603(18)$ & $-0.0096(16)$ & $0.0028(14)$ & $-0.0189(16)$ \\
\hline $\mathrm{C} 9$ & $0.0476(15)$ & $0.0727(18)$ & $0.0578(17)$ & $0.0027(13)$ & $0.0086(13)$ & $-0.0064(14)$ \\
\hline $\mathrm{C} 10$ & $0.115(3)$ & $0.075(2)$ & $0.0538(18)$ & 0.0137 (19) & $0.0211(19)$ & $0.0017(16)$ \\
\hline C11 & $0.0348(12)$ & $0.0383(14)$ & $0.0496(15)$ & $0.0037(10)$ & 0.0055 (11) & $-0.0008(11)$ \\
\hline $\mathrm{C} 12$ & $0.0408(13)$ & $0.0425(14)$ & $0.0509(15)$ & $0.0101(11)$ & $0.0033(12)$ & $0.0030(12)$ \\
\hline $\mathrm{C} 13$ & $0.0398(14)$ & $0.0430(14)$ & $0.0469(14)$ & $-0.0009(11)$ & $0.0110(11)$ & $-0.0053(11)$ \\
\hline $\mathrm{C} 14$ & $0.0442(16)$ & $0.0736(19)$ & $0.0639(18)$ & $-0.0019(14)$ & $0.0076(13)$ & $-0.0123(15)$ \\
\hline $\mathrm{C} 15$ & $0.062(2)$ & $0.101(3)$ & $0.078(2)$ & $-0.037(2)$ & $0.0290(18)$ & $-0.036(2)$ \\
\hline $\mathrm{C} 16$ & $0.108(3)$ & $0.0583(19)$ & $0.068(2)$ & $-0.038(2)$ & $0.049(2)$ & $-0.0253(16)$ \\
\hline $\mathrm{C} 17$ & $0.095(2)$ & $0.0471(17)$ & $0.0617(19)$ & $-0.0013(16)$ & $0.0260(17)$ & $0.0011(14)$ \\
\hline $\mathrm{C} 18$ & $0.0515(15)$ & $0.0464(15)$ & $0.0565(16)$ & $0.0025(12)$ & $0.0112(13)$ & $-0.0004(13)$ \\
\hline C19 & $0.0658(17)$ & $0.0420(15)$ & $0.0519(16)$ & $0.0003(12)$ & $-0.0017(13)$ & $-0.0088(12)$ \\
\hline $\mathrm{C} 20$ & $0.0679(18)$ & $0.0503(15)$ & $0.0493(16)$ & $0.0090(13)$ & $0.0246(13)$ & $0.0092(12)$ \\
\hline $\mathrm{C} 21$ & $0.0662(17)$ & $0.0518(16)$ & $0.0546(16)$ & $0.0119(13)$ & $0.0231(14)$ & $0.0034(13)$ \\
\hline $\mathrm{C} 22$ & $0.0526(16)$ & $0.0437(14)$ & $0.0548(16)$ & $-0.0053(12)$ & $0.0095(12)$ & $0.0005(12)$ \\
\hline $\mathrm{C} 23$ & $0.0492(14)$ & $0.0427(14)$ & $0.0539(16)$ & $-0.0028(11)$ & $0.0123(12)$ & $-0.0019(12)$ \\
\hline $\mathrm{C} 24$ & $0.0510(14)$ & $0.0332(12)$ & $0.0514(16)$ & $-0.0025(11)$ & $0.0093(12)$ & $-0.0007(11)$ \\
\hline $\mathrm{C} 25$ & $0.0704(18)$ & $0.0520(16)$ & $0.0562(17)$ & $0.0172(14)$ & $0.0147(14)$ & 0.0009 (13) \\
\hline $\mathrm{C} 26$ & $0.077(2)$ & $0.0632(19)$ & $0.078(2)$ & $0.0246(16)$ & 0.0208 (17) & 0.0007 (17) \\
\hline $\mathrm{C} 27$ & $0.0634(18)$ & $0.0545(17)$ & $0.083(2)$ & $0.0115(14)$ & $0.0028(16)$ & $0.0106(16)$ \\
\hline $\mathrm{C} 28$ & $0.0621(17)$ & $0.0562(16)$ & $0.0572(17)$ & $-0.0057(14)$ & $0.0073(14)$ & $0.0131(14)$ \\
\hline $\mathrm{C} 29$ & $0.0474(15)$ & $0.0418(14)$ & $0.0559(17)$ & $-0.0045(11)$ & $0.0113(13)$ & $0.0020(12)$ \\
\hline C30 & $0.083(2)$ & 0.084 (2) & 0.0484 (17) & $-0.0038(16)$ & $0.0137(15)$ & $-0.0073(15)$ \\
\hline
\end{tabular}

Geometric parameters (A, $\left.{ }^{o}\right)$

\begin{tabular}{|c|c|c|c|}
\hline $\mathrm{S} 1-\mathrm{C} 12$ & $1.661(2)$ & $\mathrm{C} 8-\mathrm{H} 8 \mathrm{~A}$ & 0.98 \\
\hline $\mathrm{F} 1-\mathrm{C} 16$ & $1.364(3)$ & C9-H9A & 0.97 \\
\hline $\mathrm{O} 1-\mathrm{C} 29$ & $1.374(3)$ & C9-H9B & 0.97 \\
\hline $\mathrm{O} 1-\mathrm{C} 30$ & $1.400(3)$ & $\mathrm{C} 10-\mathrm{H} 10 \mathrm{~A}$ & 0.97 \\
\hline $\mathrm{N} 1-\mathrm{C} 12$ & $1.387(3)$ & $\mathrm{C} 10-\mathrm{H} 10 \mathrm{~B}$ & 0.97 \\
\hline $\mathrm{N} 1-\mathrm{C} 11$ & $1.390(3)$ & $\mathrm{C} 13-\mathrm{C} 18$ & $1.372(3)$ \\
\hline $\mathrm{N} 1-\mathrm{C} 13$ & $1.438(3)$ & $\mathrm{C} 13-\mathrm{C} 14$ & $1.374(3)$ \\
\hline $\mathrm{N} 3-\mathrm{C} 12$ & $1.342(3)$ & $\mathrm{C} 14-\mathrm{C} 15$ & $1.375(4)$ \\
\hline $\mathrm{N} 3-\mathrm{N} 2$ & $1.384(3)$ & $\mathrm{C} 14-\mathrm{H} 14 \mathrm{~A}$ & 0.93 \\
\hline N3-C19 & $1.482(3)$ & $\mathrm{C} 15-\mathrm{C} 16$ & $1.358(4)$ \\
\hline $\mathrm{N} 2-\mathrm{C} 11$ & $1.309(3)$ & $\mathrm{C} 15-\mathrm{H} 15 \mathrm{~A}$ & 0.93 \\
\hline $\mathrm{N} 4-\mathrm{C} 19$ & $1.425(3)$ & $\mathrm{C} 16-\mathrm{C} 17$ & $1.351(4)$ \\
\hline
\end{tabular}




\begin{tabular}{|c|c|c|c|}
\hline $\mathrm{N} 4-\mathrm{C} 23$ & $1.454(3)$ & $\mathrm{C} 17-\mathrm{C} 18$ & $1.377(3)$ \\
\hline $\mathrm{N} 4-\mathrm{C} 20$ & $1.461(3)$ & C17-H17A & 0.93 \\
\hline $\mathrm{N} 5-\mathrm{C} 24$ & $1.424(3)$ & C18-H18A & 0.93 \\
\hline N5-C21 & $1.464(3)$ & C19-H19A & 0.97 \\
\hline $\mathrm{N} 5-\mathrm{C} 22$ & $1.468(3)$ & C19-H19B & 0.97 \\
\hline $\mathrm{C} 1-\mathrm{C} 2$ & $1.528(3)$ & $\mathrm{C} 20-\mathrm{C} 21$ & $1.512(3)$ \\
\hline $\mathrm{C} 1-\mathrm{C} 6$ & $1.537(3)$ & $\mathrm{C} 20-\mathrm{H} 20 \mathrm{~A}$ & 0.97 \\
\hline $\mathrm{C} 1-\mathrm{H} 1 \mathrm{~A}$ & 0.97 & $\mathrm{C} 20-\mathrm{H} 20 \mathrm{~B}$ & 0.97 \\
\hline $\mathrm{C} 1-\mathrm{H} 1 \mathrm{~B}$ & 0.97 & $\mathrm{C} 21-\mathrm{H} 21 \mathrm{~A}$ & 0.97 \\
\hline $\mathrm{C} 2-\mathrm{C} 3$ & $1.500(4)$ & $\mathrm{C} 21-\mathrm{H} 21 \mathrm{~B}$ & 0.97 \\
\hline $\mathrm{C} 2-\mathrm{C} 7$ & $1.510(4)$ & $\mathrm{C} 22-\mathrm{C} 23$ & $1.510(3)$ \\
\hline $\mathrm{C} 2-\mathrm{H} 2 \mathrm{~A}$ & 0.98 & $\mathrm{C} 22-\mathrm{H} 22 \mathrm{~A}$ & 0.97 \\
\hline $\mathrm{C} 3-\mathrm{C} 4$ & $1.522(4)$ & $\mathrm{C} 22-\mathrm{H} 22 \mathrm{~B}$ & 0.97 \\
\hline $\mathrm{C} 3-\mathrm{H} 3 \mathrm{~A}$ & 0.97 & $\mathrm{C} 23-\mathrm{H} 23 \mathrm{~A}$ & 0.97 \\
\hline C $3-\mathrm{H} 3 \mathrm{~B}$ & 0.97 & $\mathrm{C} 23-\mathrm{H} 23 \mathrm{~B}$ & 0.97 \\
\hline $\mathrm{C} 4-\mathrm{C} 10$ & $1.511(4)$ & $\mathrm{C} 24-\mathrm{C} 25$ & $1.380(3)$ \\
\hline $\mathrm{C} 4-\mathrm{C} 5$ & $1.541(4)$ & $\mathrm{C} 24-\mathrm{C} 29$ & $1.399(3)$ \\
\hline $\mathrm{C} 4-\mathrm{H} 4 \mathrm{~A}$ & 0.98 & $\mathrm{C} 25-\mathrm{C} 26$ & $1.388(3)$ \\
\hline $\mathrm{C} 5-\mathrm{C} 6$ & $1.531(3)$ & $\mathrm{C} 25-\mathrm{H} 25 \mathrm{~A}$ & 0.93 \\
\hline $\mathrm{C} 5-\mathrm{H} 5 \mathrm{~A}$ & 0.97 & $\mathrm{C} 26-\mathrm{C} 27$ & $1.367(4)$ \\
\hline C5-H5B & 0.97 & $\mathrm{C} 26-\mathrm{H} 26 \mathrm{~A}$ & 0.93 \\
\hline $\mathrm{C} 6-\mathrm{C} 11$ & $1.507(3)$ & $\mathrm{C} 27-\mathrm{C} 28$ & $1.385(4)$ \\
\hline $\mathrm{C} 6-\mathrm{C} 9$ & $1.538(3)$ & C27-H27A & 0.93 \\
\hline $\mathrm{C} 7-\mathrm{C} 8$ & $1.509(4)$ & $\mathrm{C} 28-\mathrm{C} 29$ & $1.387(3)$ \\
\hline C7-H7A & 0.97 & $\mathrm{C} 28-\mathrm{H} 28 \mathrm{~A}$ & 0.93 \\
\hline C7-H7B & 0.97 & $\mathrm{C} 30-\mathrm{H} 30 \mathrm{~A}$ & 0.96 \\
\hline $\mathrm{C} 8-\mathrm{C} 10$ & $1.514(4)$ & $\mathrm{C} 30-\mathrm{H} 30 \mathrm{~B}$ & 0.96 \\
\hline $\mathrm{C} 8-\mathrm{C} 9$ & $1.536(3)$ & $\mathrm{C} 30-\mathrm{H} 30 \mathrm{C}$ & 0.96 \\
\hline $\mathrm{C} 29-\mathrm{O} 1-\mathrm{C} 30$ & $118.5(2)$ & $\mathrm{N} 1-\mathrm{C} 11-\mathrm{C} 6$ & $127.1(2)$ \\
\hline $\mathrm{C} 12-\mathrm{N} 1-\mathrm{C} 11$ & $108.77(18)$ & $\mathrm{N} 3-\mathrm{C} 12-\mathrm{N} 1$ & 103.03 (19) \\
\hline $\mathrm{C} 12-\mathrm{N} 1-\mathrm{C} 13$ & $119.51(19)$ & $\mathrm{N} 3-\mathrm{C} 12-\mathrm{S} 1$ & $128.71(18)$ \\
\hline $\mathrm{C} 11-\mathrm{N} 1-\mathrm{C} 13$ & $131.62(19)$ & $\mathrm{N} 1-\mathrm{C} 12-\mathrm{S} 1$ & $128.26(18)$ \\
\hline $\mathrm{C} 12-\mathrm{N} 3-\mathrm{N} 2$ & $113.44(18)$ & $\mathrm{C} 18-\mathrm{C} 13-\mathrm{C} 14$ & $120.0(2)$ \\
\hline $\mathrm{C} 12-\mathrm{N} 3-\mathrm{C} 19$ & $125.4(2)$ & $\mathrm{C} 18-\mathrm{C} 13-\mathrm{N} 1$ & $119.7(2)$ \\
\hline $\mathrm{N} 2-\mathrm{N} 3-\mathrm{C} 19$ & $121.01(19)$ & $\mathrm{C} 14-\mathrm{C} 13-\mathrm{N} 1$ & $119.8(2)$ \\
\hline $\mathrm{C} 11-\mathrm{N} 2-\mathrm{N} 3$ & $105.01(19)$ & $\mathrm{C} 13-\mathrm{C} 14-\mathrm{C} 15$ & $119.9(3)$ \\
\hline $\mathrm{C} 19-\mathrm{N} 4-\mathrm{C} 23$ & $114.40(19)$ & $\mathrm{C} 13-\mathrm{C} 14-\mathrm{H} 14 \mathrm{~A}$ & 120.1 \\
\hline $\mathrm{C} 19-\mathrm{N} 4-\mathrm{C} 20$ & $113.73(19)$ & $\mathrm{C} 15-\mathrm{C} 14-\mathrm{H} 14 \mathrm{~A}$ & 120.1 \\
\hline $\mathrm{C} 23-\mathrm{N} 4-\mathrm{C} 20$ & $110.29(18)$ & $\mathrm{C} 16-\mathrm{C} 15-\mathrm{C} 14$ & $118.4(3)$ \\
\hline $\mathrm{C} 24-\mathrm{N} 5-\mathrm{C} 21$ & $116.26(18)$ & $\mathrm{C} 16-\mathrm{C} 15-\mathrm{H} 15 \mathrm{~A}$ & 120.8 \\
\hline $\mathrm{C} 24-\mathrm{N} 5-\mathrm{C} 22$ & $113.26(18)$ & $\mathrm{C} 14-\mathrm{C} 15-\mathrm{H} 15 \mathrm{~A}$ & 120.8 \\
\hline $\mathrm{C} 21-\mathrm{N} 5-\mathrm{C} 22$ & $109.82(19)$ & $\mathrm{C} 17-\mathrm{C} 16-\mathrm{C} 15$ & $123.3(3)$ \\
\hline $\mathrm{C} 2-\mathrm{C} 1-\mathrm{C} 6$ & $109.8(2)$ & $\mathrm{C} 17-\mathrm{C} 16-\mathrm{F} 1$ & $118.6(4)$ \\
\hline $\mathrm{C} 2-\mathrm{C} 1-\mathrm{H} 1 \mathrm{~A}$ & 109.7 & $\mathrm{C} 15-\mathrm{C} 16-\mathrm{F} 1$ & $118.0(3)$ \\
\hline $\mathrm{C} 6-\mathrm{C} 1-\mathrm{H} 1 \mathrm{~A}$ & 109.7 & $\mathrm{C} 16-\mathrm{C} 17-\mathrm{C} 18$ & $118.0(3)$ \\
\hline $\mathrm{C} 2-\mathrm{C} 1-\mathrm{H} 1 \mathrm{~B}$ & 109.7 & $\mathrm{C} 16-\mathrm{C} 17-\mathrm{H} 17 \mathrm{~A}$ & 121 \\
\hline $\mathrm{C} 6-\mathrm{C} 1-\mathrm{H} 1 \mathrm{~B}$ & 109.7 & $\mathrm{C} 18-\mathrm{C} 17-\mathrm{H} 17 \mathrm{~A}$ & 121 \\
\hline
\end{tabular}




\begin{tabular}{|c|c|c|c|}
\hline $\mathrm{H} 1 \mathrm{~A}-\mathrm{C} 1-\mathrm{H} 1 \mathrm{~B}$ & 108.2 & $\mathrm{C} 13-\mathrm{C} 18-\mathrm{C} 17$ & $120.3(3)$ \\
\hline $\mathrm{C} 3-\mathrm{C} 2-\mathrm{C} 7$ & $109.5(2)$ & $\mathrm{C} 13-\mathrm{C} 18-\mathrm{H} 18 \mathrm{~A}$ & 119.8 \\
\hline $\mathrm{C} 3-\mathrm{C} 2-\mathrm{C} 1$ & $110.6(2)$ & $\mathrm{C} 17-\mathrm{C} 18-\mathrm{H} 18 \mathrm{~A}$ & 119.8 \\
\hline $\mathrm{C} 7-\mathrm{C} 2-\mathrm{C} 1$ & $109.6(2)$ & $\mathrm{N} 4-\mathrm{C} 19-\mathrm{N} 3$ & $115.62(19)$ \\
\hline $\mathrm{C} 3-\mathrm{C} 2-\mathrm{H} 2 \mathrm{~A}$ & 109 & $\mathrm{~N} 4-\mathrm{C} 19-\mathrm{H} 19 \mathrm{~A}$ & 108.4 \\
\hline $\mathrm{C} 7-\mathrm{C} 2-\mathrm{H} 2 \mathrm{~A}$ & 109 & $\mathrm{~N} 3-\mathrm{C} 19-\mathrm{H} 19 \mathrm{~A}$ & 108.4 \\
\hline $\mathrm{C} 1-\mathrm{C} 2-\mathrm{H} 2 \mathrm{~A}$ & 109 & $\mathrm{~N} 4-\mathrm{C} 19-\mathrm{H} 19 \mathrm{~B}$ & 108.4 \\
\hline $\mathrm{C} 2-\mathrm{C} 3-\mathrm{C} 4$ & $109.2(2)$ & N3-C19-H19B & 108.4 \\
\hline $\mathrm{C} 2-\mathrm{C} 3-\mathrm{H} 3 \mathrm{~A}$ & 109.8 & $\mathrm{H} 19 \mathrm{~A}-\mathrm{C} 19-\mathrm{H} 19 \mathrm{~B}$ & 107.4 \\
\hline $\mathrm{C} 4-\mathrm{C} 3-\mathrm{H} 3 \mathrm{~A}$ & 109.8 & $\mathrm{~N} 4-\mathrm{C} 20-\mathrm{C} 21$ & $111.4(2)$ \\
\hline $\mathrm{C} 2-\mathrm{C} 3-\mathrm{H} 3 \mathrm{~B}$ & 109.8 & $\mathrm{~N} 4-\mathrm{C} 20-\mathrm{H} 20 \mathrm{~A}$ & 109.4 \\
\hline $\mathrm{C} 4-\mathrm{C} 3-\mathrm{H} 3 \mathrm{~B}$ & 109.8 & $\mathrm{C} 21-\mathrm{C} 20-\mathrm{H} 20 \mathrm{~A}$ & 109.4 \\
\hline $\mathrm{H} 3 \mathrm{~A}-\mathrm{C} 3-\mathrm{H} 3 \mathrm{~B}$ & 108.3 & $\mathrm{~N} 4-\mathrm{C} 20-\mathrm{H} 20 \mathrm{~B}$ & 109.4 \\
\hline $\mathrm{C} 10-\mathrm{C} 4-\mathrm{C} 3$ & $110.0(2)$ & $\mathrm{C} 21-\mathrm{C} 20-\mathrm{H} 20 \mathrm{~B}$ & 109.4 \\
\hline $\mathrm{C} 10-\mathrm{C} 4-\mathrm{C} 5$ & $109.3(2)$ & $\mathrm{H} 20 \mathrm{~A}-\mathrm{C} 20-\mathrm{H} 20 \mathrm{~B}$ & 108 \\
\hline $\mathrm{C} 3-\mathrm{C} 4-\mathrm{C} 5$ & $109.2(3)$ & $\mathrm{N} 5-\mathrm{C} 21-\mathrm{C} 20$ & $110.12(19)$ \\
\hline $\mathrm{C} 10-\mathrm{C} 4-\mathrm{H} 4 \mathrm{~A}$ & 109.4 & $\mathrm{~N} 5-\mathrm{C} 21-\mathrm{H} 21 \mathrm{~A}$ & 109.6 \\
\hline $\mathrm{C} 3-\mathrm{C} 4-\mathrm{H} 4 \mathrm{~A}$ & 109.4 & $\mathrm{C} 20-\mathrm{C} 21-\mathrm{H} 21 \mathrm{~A}$ & 109.6 \\
\hline $\mathrm{C} 5-\mathrm{C} 4-\mathrm{H} 4 \mathrm{~A}$ & 109.4 & $\mathrm{~N} 5-\mathrm{C} 21-\mathrm{H} 21 \mathrm{~B}$ & 109.6 \\
\hline $\mathrm{C} 6-\mathrm{C} 5-\mathrm{C} 4$ & $110.3(2)$ & $\mathrm{C} 20-\mathrm{C} 21-\mathrm{H} 21 \mathrm{~B}$ & 109.6 \\
\hline $\mathrm{C} 6-\mathrm{C} 5-\mathrm{H} 5 \mathrm{~A}$ & 109.6 & $\mathrm{H} 21 \mathrm{~A}-\mathrm{C} 21-\mathrm{H} 21 \mathrm{~B}$ & 108.2 \\
\hline $\mathrm{C} 4-\mathrm{C} 5-\mathrm{H} 5 \mathrm{~A}$ & 109.6 & $\mathrm{~N} 5-\mathrm{C} 22-\mathrm{C} 23$ & $109.88(18)$ \\
\hline $\mathrm{C} 6-\mathrm{C} 5-\mathrm{H} 5 \mathrm{~B}$ & 109.6 & $\mathrm{~N} 5-\mathrm{C} 22-\mathrm{H} 22 \mathrm{~A}$ & 109.7 \\
\hline $\mathrm{C} 4-\mathrm{C} 5-\mathrm{H} 5 \mathrm{~B}$ & 109.6 & $\mathrm{C} 23-\mathrm{C} 22-\mathrm{H} 22 \mathrm{~A}$ & 109.7 \\
\hline $\mathrm{H} 5 \mathrm{~A}-\mathrm{C} 5-\mathrm{H} 5 \mathrm{~B}$ & 108.1 & N5-C22-H22B & 109.7 \\
\hline $\mathrm{C} 11-\mathrm{C} 6-\mathrm{C} 5$ & $108.80(19)$ & $\mathrm{C} 23-\mathrm{C} 22-\mathrm{H} 22 \mathrm{~B}$ & 109.7 \\
\hline $\mathrm{C} 11-\mathrm{C} 6-\mathrm{C} 1$ & $111.76(19)$ & $\mathrm{H} 22 \mathrm{~A}-\mathrm{C} 22-\mathrm{H} 22 \mathrm{~B}$ & 108.2 \\
\hline $\mathrm{C} 5-\mathrm{C} 6-\mathrm{C} 1$ & $108.2(2)$ & $\mathrm{N} 4-\mathrm{C} 23-\mathrm{C} 22$ & $109.52(19)$ \\
\hline $\mathrm{C} 11-\mathrm{C} 6-\mathrm{C} 9$ & $110.99(18)$ & $\mathrm{N} 4-\mathrm{C} 23-\mathrm{H} 23 \mathrm{~A}$ & 109.8 \\
\hline $\mathrm{C} 5-\mathrm{C} 6-\mathrm{C} 9$ & $108.4(2)$ & $\mathrm{C} 22-\mathrm{C} 23-\mathrm{H} 23 \mathrm{~A}$ & 109.8 \\
\hline $\mathrm{C} 1-\mathrm{C} 6-\mathrm{C} 9$ & $108.60(19)$ & $\mathrm{N} 4-\mathrm{C} 23-\mathrm{H} 23 \mathrm{~B}$ & 109.8 \\
\hline $\mathrm{C} 8-\mathrm{C} 7-\mathrm{C} 2$ & $109.8(2)$ & $\mathrm{C} 22-\mathrm{C} 23-\mathrm{H} 23 \mathrm{~B}$ & 109.8 \\
\hline $\mathrm{C} 8-\mathrm{C} 7-\mathrm{H} 7 \mathrm{~A}$ & 109.7 & $\mathrm{H} 23 \mathrm{~A}-\mathrm{C} 23-\mathrm{H} 23 \mathrm{~B}$ & 108.2 \\
\hline $\mathrm{C} 2-\mathrm{C} 7-\mathrm{H} 7 \mathrm{~A}$ & 109.7 & $\mathrm{C} 25-\mathrm{C} 24-\mathrm{C} 29$ & $117.8(2)$ \\
\hline $\mathrm{C} 8-\mathrm{C} 7-\mathrm{H} 7 \mathrm{~B}$ & 109.7 & $\mathrm{C} 25-\mathrm{C} 24-\mathrm{N} 5$ & $123.5(2)$ \\
\hline $\mathrm{C} 2-\mathrm{C} 7-\mathrm{H} 7 \mathrm{~B}$ & 109.7 & $\mathrm{C} 29-\mathrm{C} 24-\mathrm{N} 5$ & $118.7(2)$ \\
\hline $\mathrm{H} 7 \mathrm{~A}-\mathrm{C} 7-\mathrm{H} 7 \mathrm{~B}$ & 108.2 & $\mathrm{C} 24-\mathrm{C} 25-\mathrm{C} 26$ & $121.4(3)$ \\
\hline $\mathrm{C} 7-\mathrm{C} 8-\mathrm{C} 10$ & $110.0(2)$ & $\mathrm{C} 24-\mathrm{C} 25-\mathrm{H} 25 \mathrm{~A}$ & 119.3 \\
\hline $\mathrm{C} 7-\mathrm{C} 8-\mathrm{C} 9$ & $109.2(2)$ & $\mathrm{C} 26-\mathrm{C} 25-\mathrm{H} 25 \mathrm{~A}$ & 119.3 \\
\hline $\mathrm{C} 10-\mathrm{C} 8-\mathrm{C} 9$ & $109.4(2)$ & $\mathrm{C} 27-\mathrm{C} 26-\mathrm{C} 25$ & $120.3(3)$ \\
\hline $\mathrm{C} 7-\mathrm{C} 8-\mathrm{H} 8 \mathrm{~A}$ & 109.4 & $\mathrm{C} 27-\mathrm{C} 26-\mathrm{H} 26 \mathrm{~A}$ & 119.9 \\
\hline $\mathrm{C} 10-\mathrm{C} 8-\mathrm{H} 8 \mathrm{~A}$ & 109.4 & $\mathrm{C} 25-\mathrm{C} 26-\mathrm{H} 26 \mathrm{~A}$ & 119.9 \\
\hline $\mathrm{C} 9-\mathrm{C} 8-\mathrm{H} 8 \mathrm{~A}$ & 109.4 & $\mathrm{C} 26-\mathrm{C} 27-\mathrm{C} 28$ & $119.5(3)$ \\
\hline $\mathrm{C} 8-\mathrm{C} 9-\mathrm{C} 6$ & $110.0(2)$ & $\mathrm{C} 26-\mathrm{C} 27-\mathrm{H} 27 \mathrm{~A}$ & 120.2 \\
\hline $\mathrm{C} 8-\mathrm{C} 9-\mathrm{H} 9 \mathrm{~A}$ & 109.7 & $\mathrm{C} 28-\mathrm{C} 27-\mathrm{H} 27 \mathrm{~A}$ & 120.2 \\
\hline C6- $69-\mathrm{H} 9 \mathrm{~A}$ & 109.7 & $\mathrm{C} 27-\mathrm{C} 28-\mathrm{C} 29$ & $120.2(3)$ \\
\hline $\mathrm{C} 8-\mathrm{C} 9-\mathrm{H} 9 \mathrm{~B}$ & 109.7 & $\mathrm{C} 27-\mathrm{C} 28-\mathrm{H} 28 \mathrm{~A}$ & 119.9 \\
\hline C6-C9-H9B & 109.7 & $\mathrm{C} 29-\mathrm{C} 28-\mathrm{H} 28 \mathrm{~A}$ & 119.9 \\
\hline
\end{tabular}




\begin{tabular}{|c|c|c|c|}
\hline $\mathrm{H} 9 \mathrm{~A}-\mathrm{C} 9-\mathrm{H} 9 \mathrm{~B}$ & 108.2 & $\mathrm{O} 1-\mathrm{C} 29-\mathrm{C} 28$ & $123.7(2)$ \\
\hline $\mathrm{C} 4-\mathrm{C} 10-\mathrm{C} 8$ & $109.2(2)$ & $\mathrm{O} 1-\mathrm{C} 29-\mathrm{C} 24$ & $115.7(2)$ \\
\hline $\mathrm{C} 4-\mathrm{C} 10-\mathrm{H} 10 \mathrm{~A}$ & 109.8 & $\mathrm{C} 28-\mathrm{C} 29-\mathrm{C} 24$ & $120.6(2)$ \\
\hline $\mathrm{C} 8-\mathrm{C} 10-\mathrm{H} 10 \mathrm{~A}$ & 109.8 & $\mathrm{O} 1-\mathrm{C} 30-\mathrm{H} 30 \mathrm{~A}$ & 109.5 \\
\hline $\mathrm{C} 4-\mathrm{C} 10-\mathrm{H} 10 \mathrm{~B}$ & 109.8 & $\mathrm{O} 1-\mathrm{C} 30-\mathrm{H} 30 \mathrm{~B}$ & 109.5 \\
\hline $\mathrm{C} 8-\mathrm{C} 10-\mathrm{H} 10 \mathrm{~B}$ & 109.8 & $\mathrm{H} 30 \mathrm{~A}-\mathrm{C} 30-\mathrm{H} 30 \mathrm{~B}$ & 109.5 \\
\hline $\mathrm{H} 10 \mathrm{~A}-\mathrm{C} 10-\mathrm{H} 10 \mathrm{~B}$ & 108.3 & $\mathrm{O} 1-\mathrm{C} 30-\mathrm{H} 30 \mathrm{C}$ & 109.5 \\
\hline $\mathrm{N} 2-\mathrm{C} 11-\mathrm{N} 1$ & 109.73 (19) & $\mathrm{H} 30 \mathrm{~A}-\mathrm{C} 30-\mathrm{H} 30 \mathrm{C}$ & 109.5 \\
\hline $\mathrm{N} 2-\mathrm{C} 11-\mathrm{C} 6$ & $123.1(2)$ & $\mathrm{H} 30 \mathrm{~B}-\mathrm{C} 30-\mathrm{H} 30 \mathrm{C}$ & 109.5 \\
\hline $\mathrm{C} 12-\mathrm{N} 3-\mathrm{N} 2-\mathrm{C} 11$ & $0.7(2)$ & $\mathrm{C} 11-\mathrm{N} 1-\mathrm{C} 12-\mathrm{S} 1$ & $-178.65(17)$ \\
\hline $\mathrm{C} 19-\mathrm{N} 3-\mathrm{N} 2-\mathrm{C} 11$ & $176.39(19)$ & $\mathrm{C} 13-\mathrm{N} 1-\mathrm{C} 12-\mathrm{S} 1$ & $4.6(3)$ \\
\hline $\mathrm{C} 6-\mathrm{C} 1-\mathrm{C} 2-\mathrm{C} 3$ & $-60.5(3)$ & $\mathrm{C} 12-\mathrm{N} 1-\mathrm{C} 13-\mathrm{C} 18$ & $88.6(3)$ \\
\hline $\mathrm{C} 6-\mathrm{C} 1-\mathrm{C} 2-\mathrm{C} 7$ & $60.3(3)$ & $\mathrm{C} 11-\mathrm{N} 1-\mathrm{C} 13-\mathrm{C} 18$ & $-87.3(3)$ \\
\hline $\mathrm{C} 7-\mathrm{C} 2-\mathrm{C} 3-\mathrm{C} 4$ & $-60.2(3)$ & $\mathrm{C} 12-\mathrm{N} 1-\mathrm{C} 13-\mathrm{C} 14$ & $-83.7(3)$ \\
\hline $\mathrm{C} 1-\mathrm{C} 2-\mathrm{C} 3-\mathrm{C} 4$ & $60.6(3)$ & $\mathrm{C} 11-\mathrm{N} 1-\mathrm{C} 13-\mathrm{C} 14$ & $100.5(3)$ \\
\hline $\mathrm{C} 2-\mathrm{C} 3-\mathrm{C} 4-\mathrm{C} 10$ & $60.1(3)$ & $\mathrm{C} 18-\mathrm{C} 13-\mathrm{C} 14-\mathrm{C} 15$ & $-0.7(4)$ \\
\hline $\mathrm{C} 2-\mathrm{C} 3-\mathrm{C} 4-\mathrm{C} 5$ & $-59.9(3)$ & $\mathrm{N} 1-\mathrm{C} 13-\mathrm{C} 14-\mathrm{C} 15$ & $171.5(2)$ \\
\hline $\mathrm{C} 10-\mathrm{C} 4-\mathrm{C} 5-\mathrm{C} 6$ & $-60.3(3)$ & $\mathrm{C} 13-\mathrm{C} 14-\mathrm{C} 15-\mathrm{C} 16$ & $-0.2(4)$ \\
\hline $\mathrm{C} 3-\mathrm{C} 4-\mathrm{C} 5-\mathrm{C} 6$ & $60.2(3)$ & $\mathrm{C} 14-\mathrm{C} 15-\mathrm{C} 16-\mathrm{C} 17$ & $1.5(4)$ \\
\hline $\mathrm{C} 4-\mathrm{C} 5-\mathrm{C} 6-\mathrm{C} 11$ & $179.3(2)$ & $\mathrm{C} 14-\mathrm{C} 15-\mathrm{C} 16-\mathrm{F} 1$ & $-176.4(2)$ \\
\hline $\mathrm{C} 4-\mathrm{C} 5-\mathrm{C} 6-\mathrm{C} 1$ & $-59.1(3)$ & $\mathrm{C} 15-\mathrm{C} 16-\mathrm{C} 17-\mathrm{C} 18$ & $-2.0(4)$ \\
\hline $\mathrm{C} 4-\mathrm{C} 5-\mathrm{C} 6-\mathrm{C} 9$ & $58.5(3)$ & $\mathrm{F} 1-\mathrm{C} 16-\mathrm{C} 17-\mathrm{C} 18$ & $176.0(2)$ \\
\hline $\mathrm{C} 2-\mathrm{C} 1-\mathrm{C} 6-\mathrm{C} 11$ & $178.4(2)$ & $\mathrm{C} 14-\mathrm{C} 13-\mathrm{C} 18-\mathrm{C} 17$ & $0.3(4)$ \\
\hline $\mathrm{C} 2-\mathrm{C} 1-\mathrm{C} 6-\mathrm{C} 5$ & $58.6(3)$ & $\mathrm{N} 1-\mathrm{C} 13-\mathrm{C} 18-\mathrm{C} 17$ & $-172.0(2)$ \\
\hline $\mathrm{C} 2-\mathrm{C} 1-\mathrm{C} 6-\mathrm{C} 9$ & $-58.8(3)$ & $\mathrm{C} 16-\mathrm{C} 17-\mathrm{C} 18-\mathrm{C} 13$ & $1.0(4)$ \\
\hline $\mathrm{C} 3-\mathrm{C} 2-\mathrm{C} 7-\mathrm{C} 8$ & $60.3(3)$ & $\mathrm{C} 23-\mathrm{N} 4-\mathrm{C} 19-\mathrm{N} 3$ & $75.5(3)$ \\
\hline $\mathrm{C} 1-\mathrm{C} 2-\mathrm{C} 7-\mathrm{C} 8$ & $-61.2(3)$ & $\mathrm{C} 20-\mathrm{N} 4-\mathrm{C} 19-\mathrm{N} 3$ & $-52.4(3)$ \\
\hline $\mathrm{C} 2-\mathrm{C} 7-\mathrm{C} 8-\mathrm{C} 10$ & $-59.4(3)$ & $\mathrm{C} 12-\mathrm{N} 3-\mathrm{C} 19-\mathrm{N} 4$ & $104.2(3)$ \\
\hline $\mathrm{C} 2-\mathrm{C} 7-\mathrm{C} 8-\mathrm{C} 9$ & $60.7(3)$ & $\mathrm{N} 2-\mathrm{N} 3-\mathrm{C} 19-\mathrm{N} 4$ & $-71.0(3)$ \\
\hline $\mathrm{C} 7-\mathrm{C} 8-\mathrm{C} 9-\mathrm{C} 6$ & $-59.9(3)$ & $\mathrm{C} 19-\mathrm{N} 4-\mathrm{C} 20-\mathrm{C} 21$ & $-172.94(19)$ \\
\hline $\mathrm{C} 10-\mathrm{C} 8-\mathrm{C} 9-\mathrm{C} 6$ & $60.6(3)$ & $\mathrm{C} 23-\mathrm{N} 4-\mathrm{C} 20-\mathrm{C} 21$ & $57.0(2)$ \\
\hline $\mathrm{C} 11-\mathrm{C} 6-\mathrm{C} 9-\mathrm{C} 8$ & $-178.1(2)$ & $\mathrm{C} 24-\mathrm{N} 5-\mathrm{C} 21-\mathrm{C} 20$ & $-172.6(2)$ \\
\hline $\mathrm{C} 5-\mathrm{C} 6-\mathrm{C} 9-\mathrm{C} 8$ & $-58.6(3)$ & $\mathrm{C} 22-\mathrm{N} 5-\mathrm{C} 21-\mathrm{C} 20$ & $57.1(3)$ \\
\hline $\mathrm{C} 1-\mathrm{C} 6-\mathrm{C} 9-\mathrm{C} 8$ & $58.7(3)$ & $\mathrm{N} 4-\mathrm{C} 20-\mathrm{C} 21-\mathrm{N} 5$ & $-56.0(3)$ \\
\hline $\mathrm{C} 3-\mathrm{C} 4-\mathrm{C} 10-\mathrm{C} 8$ & $-59.0(3)$ & $\mathrm{C} 24-\mathrm{N} 5-\mathrm{C} 22-\mathrm{C} 23$ & $168.36(19)$ \\
\hline $\mathrm{C} 5-\mathrm{C} 4-\mathrm{C} 10-\mathrm{C} 8$ & $61.0(3)$ & $\mathrm{C} 21-\mathrm{N} 5-\mathrm{C} 22-\mathrm{C} 23$ & $-59.8(2)$ \\
\hline $\mathrm{C} 7-\mathrm{C} 8-\mathrm{C} 10-\mathrm{C} 4$ & $58.6(3)$ & $\mathrm{C} 19-\mathrm{N} 4-\mathrm{C} 23-\mathrm{C} 22$ & 171.58 (19) \\
\hline $\mathrm{C} 9-\mathrm{C} 8-\mathrm{C} 10-\mathrm{C} 4$ & $-61.4(3)$ & $\mathrm{C} 20-\mathrm{N} 4-\mathrm{C} 23-\mathrm{C} 22$ & $-58.7(2)$ \\
\hline $\mathrm{N} 3-\mathrm{N} 2-\mathrm{C} 11-\mathrm{N} 1$ & $0.3(2)$ & $\mathrm{N} 5-\mathrm{C} 22-\mathrm{C} 23-\mathrm{N} 4$ & $60.5(2)$ \\
\hline N3-N2-C11-C6 & $-177.88(18)$ & $\mathrm{C} 21-\mathrm{N} 5-\mathrm{C} 24-\mathrm{C} 25$ & $-13.9(3)$ \\
\hline $\mathrm{C} 12-\mathrm{N} 1-\mathrm{C} 11-\mathrm{N} 2$ & $-1.1(2)$ & $\mathrm{C} 22-\mathrm{N} 5-\mathrm{C} 24-\mathrm{C} 25$ & $114.7(3)$ \\
\hline $\mathrm{C} 13-\mathrm{N} 1-\mathrm{C} 11-\mathrm{N} 2$ & $175.1(2)$ & $\mathrm{C} 21-\mathrm{N} 5-\mathrm{C} 24-\mathrm{C} 29$ & $165.9(2)$ \\
\hline $\mathrm{C} 12-\mathrm{N} 1-\mathrm{C} 11-\mathrm{C} 6$ & $177.0(2)$ & $\mathrm{C} 22-\mathrm{N} 5-\mathrm{C} 24-\mathrm{C} 29$ & $-65.5(3)$ \\
\hline $\mathrm{C} 13-\mathrm{N} 1-\mathrm{C} 11-\mathrm{C} 6$ & $-6.8(4)$ & $\mathrm{C} 29-\mathrm{C} 24-\mathrm{C} 25-\mathrm{C} 26$ & $3.0(4)$ \\
\hline $\mathrm{C} 5-\mathrm{C} 6-\mathrm{C} 11-\mathrm{N} 2$ & $-3.9(3)$ & $\mathrm{N} 5-\mathrm{C} 24-\mathrm{C} 25-\mathrm{C} 26$ & $-177.2(2)$ \\
\hline $\mathrm{C} 1-\mathrm{C} 6-\mathrm{C} 11-\mathrm{N} 2$ & $-123.3(2)$ & $\mathrm{C} 24-\mathrm{C} 25-\mathrm{C} 26-\mathrm{C} 27$ & $0.6(4)$ \\
\hline $\mathrm{C} 9-\mathrm{C} 6-\mathrm{C} 11-\mathrm{N} 2$ & $115.2(2)$ & $\mathrm{C} 25-\mathrm{C} 26-\mathrm{C} 27-\mathrm{C} 28$ & $-3.1(4)$ \\
\hline
\end{tabular}




$\begin{array}{llll}\mathrm{C} 5-\mathrm{C} 6-\mathrm{C} 11-\mathrm{N} 1 & 178.3(2) & \mathrm{C} 26-\mathrm{C} 27-\mathrm{C} 28-\mathrm{C} 29 & 2.0(4) \\ \mathrm{C} 1-\mathrm{C} 6-\mathrm{C} 11-\mathrm{N} 1 & 58.8(3) & \mathrm{C} 30-\mathrm{O} 1-\mathrm{C} 29-\mathrm{C} 28 & -2.9(3) \\ \mathrm{C} 9-\mathrm{C} 6-\mathrm{C} 11-\mathrm{N} 1 & -62.6(3) & \mathrm{C} 30-\mathrm{O} 1-\mathrm{C} 29-\mathrm{C} 24 & 176.8(2) \\ \mathrm{N} 2-\mathrm{N} 3-\mathrm{C} 12-\mathrm{N} 1 & -1.3(2) & \mathrm{C} 27-\mathrm{C} 28-\mathrm{C} 29-\mathrm{O} 1 & -178.5(2) \\ \mathrm{C} 19-\mathrm{N} 3-\mathrm{C} 12-\mathrm{N} 1 & -176.79(19) & \mathrm{C} 27-\mathrm{C} 28-\mathrm{C} 29-\mathrm{C} 24 & 1.7(4) \\ \mathrm{N} 2-\mathrm{N} 3-\mathrm{C} 12-\mathrm{S} 1 & 178.75(16) & \mathrm{C} 25-\mathrm{C} 24-\mathrm{C} 29-\mathrm{O} 1 & 176.1(2) \\ \mathrm{C} 19-\mathrm{N} 3-\mathrm{C} 12-\mathrm{S} 1 & 3.3(3) & \mathrm{N} 5-\mathrm{C} 24-\mathrm{C} 29-\mathrm{O} 1 & -3.7(3) \\ \mathrm{C} 11-\mathrm{N} 1-\mathrm{C} 12-\mathrm{N} 3 & 1.4(2) & \mathrm{C} 25-\mathrm{C} 24-\mathrm{C} 29-\mathrm{C} 28 & -4.2(3) \\ \mathrm{C} 13-\mathrm{N} 1-\mathrm{C} 12-\mathrm{N} 3 & -175.31(18) & \mathrm{N} 5-\mathrm{C} 24-\mathrm{C} 29-\mathrm{C} 28 & 176.1(2)\end{array}$

Hydrogen-bond geometry $\left(A,{ }^{\circ}\right)$

$\mathrm{Cg} 1$ and $\mathrm{Cg} 8$ are the centroids of rings $(\mathrm{N} 1-\mathrm{N} 3 / \mathrm{C} 11 / \mathrm{C} 12)$ and $(\mathrm{C} 24-\mathrm{C} 29)$, respectively.

\begin{tabular}{lllll}
\hline$D-\mathrm{H} \cdots A$ & $D-\mathrm{H}$ & $\mathrm{H} \cdots A$ & $D \cdots A$ & $D-\mathrm{H} \cdots A$ \\
\hline $\mathrm{C} 21-\mathrm{H} 21 A \cdots \mathrm{F} 1^{\mathrm{i}}$ & 0.97 & 2.47 & $3.407(3)$ & 162 \\
$\mathrm{C} 18-\mathrm{H} 18 A \cdots C g 1^{\mathrm{ii}}$ & 0.93 & 2.81 & 3.661 & 152 \\
$\mathrm{C} 9-\mathrm{H} 9 A \cdots C g 8^{\mathrm{iii}}$ & 0.97 & 2.80 & 3.697 & 155 \\
\hline
\end{tabular}

Symmetry codes: (i) $x, y-1, z$; (ii) $-x,-y,-z$; (iii) $-x+1, y-1 / 2,-z+1 / 2$. 